\title{
Structure-property relationships of microphase-separated metallosupramolecular polymers
}

\section{Electronic Supplementary Information}

Laura N. Neumann, ${ }^{+}$Ilja Gunkel, ${ }^{+}$Amber Barron, ${ }^{+}$Emad Oveisi, ${ }^{\ddagger}$ Albrecht Petzold,,$\$$ Thomas Thurn-Albrecht, ${ }^{\S}, *$ Stephen Schrettl, ${ }^{+, *}$ Christoph Weder ${ }^{+}, *$

+ Adolphe Merkle Institute (AMI), University of Fribourg, Chemin des Verdiers 4, 1700 Fribourg, Switzerland.

‡ Interdisciplinary Centre for Electron Microscopy (CIME), Ecole Polytechnique Fédérale de Lausanne (EPFL), EPFL - SB - CIME, Bâtiment MXC-135, Station 12, CH-1015 Lausanne, Switzerland.

$\S$ Martin-Luther-Universität Halle - Wittenberg, Naturwissenschaftliche Fakultät II - Chemie und Physik, Institut für Physik, von-Danckelmann-Platz 3, D-06120 Halle (Saale), Germany.

*To whom correspondence should be addressed:

thomas.thurn-albrecht@physik.uni-halle.de stephen.schrettl@unifr.ch christoph.weder@unifr.ch 


\section{Table of Contents}

1.

1.1.

1.2 .

1.3.

1.4.

1.5.

2.

3.

3.1.

3.2 .

3.3.

3.4 .

4.

Supplementary Figures S1-S36. 2

Materials and Spectrophotometric Titrations S1-S6

2

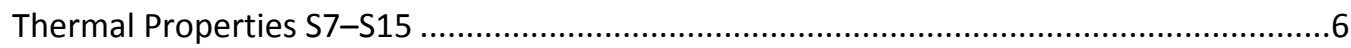

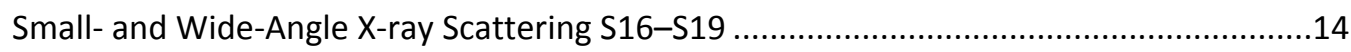

Thermomechanical Characterization S20 ........................................................16

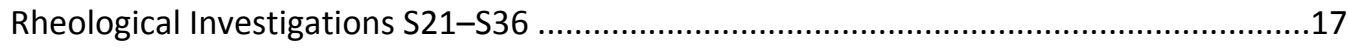

Supplementary Tables S1-S2 ...................................................27

Experimental Details................................................................28

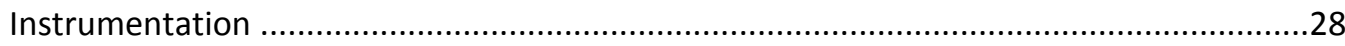

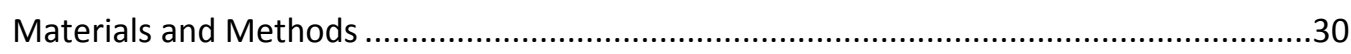

Synthetic Procedures and Analytical Data.............................................................

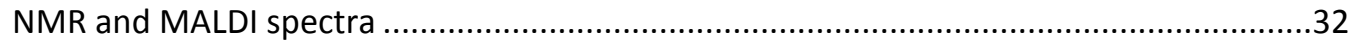

References.............................................................................36 


\section{Supplementary Figures S1-S36}

\subsection{Materials and Spectrophotometric Titrations S1-S6}
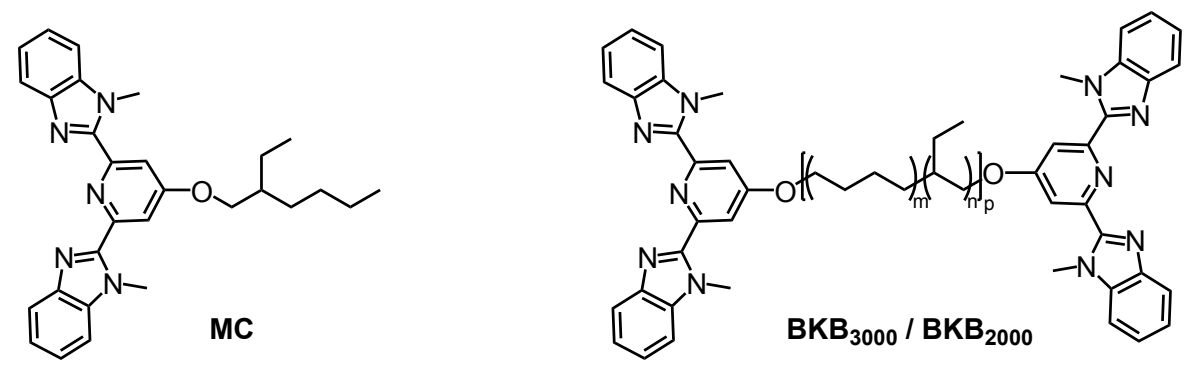

Figure S1. Chemical structures of bis(1'-methylbenzimidazoyl)-4-(2-ethylhexyloxy)pyridine (MC) that was used as a model compound and of the macromonomers that were employed as macromonomer for the preparation of the investigated metallosupramolecular polymers, i.e., BKB $_{3000}$ (with $M_{\mathrm{n}}=3800 \mathrm{~g} \mathrm{~mol}^{-1} ; \mathrm{m} \approx 0.32, \mathrm{n} \approx 0.68, \mathrm{p} \approx 55$ ) and $\mathbf{B K B}_{2000}$ (with $M_{\mathrm{n}}=3000 \mathrm{~g} \mathrm{~mol}^{-1} ; \mathrm{m} \approx 0.30, \mathrm{n} \approx 0.70, \mathrm{p} \approx 41$ ) consisting of the telechelic PEB core end-functionalized with 2,6-bis(1'-methylbenzimidazolyl)-pyridine (Mebip) ligands. The Mebip ligand, the low molecular weight model compound MC, and the BKB macromonomers were prepared following previously reported procedures. ${ }^{1,2,7}$

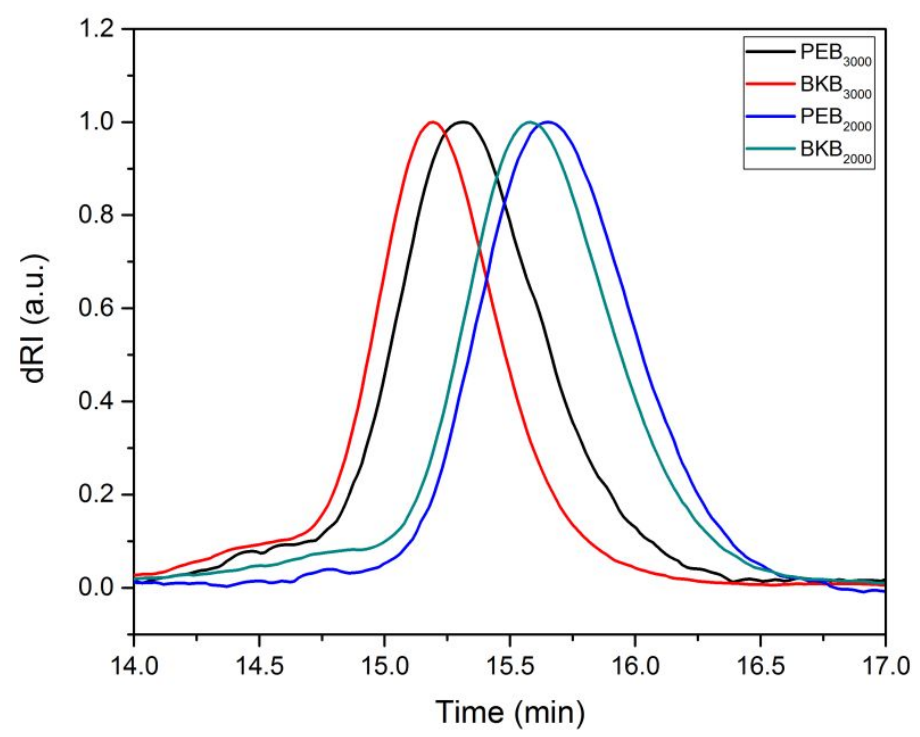

Figure S2. Comparison of the size exclusion chromatography (SEC) traces of the telechelic, bis-hydroxyl terminated poly(ethylene-co-butylene) $\mathrm{PEB}_{2000}\left(M_{\mathrm{n}}\right.$ of $\left.3100 \mathrm{~g} \mathrm{~mol}^{-1}\right)$ and $\mathrm{PEB}_{2000}\left(M_{\mathrm{n}}\right.$ of $\left.2100 \mathrm{~g} \mathrm{~mol}^{-1}\right)$, as well as of the SEC traces of the macromonomers $\mathbf{B K B}_{3000}$ (with an $M_{\mathrm{n}}=3800 \mathrm{~g} \mathrm{~mol}^{-1} ; \mathrm{m} \approx 0.32, \mathrm{n} \approx 0.68, \mathrm{p} \approx 55$ ) and $\mathbf{B K B}_{\mathbf{2 0 0 0}}$ (with an $M_{\mathrm{n}}=$ $3000 \mathrm{~g} \mathrm{~mol}^{-1} ; \mathrm{m} \approx 0.30, \mathrm{n} \approx 0.70, \mathrm{p} \approx 41$ ) consisting of the telechelic PEB core end-functionalized with 2,6-bis(1'methylbenzimidazolyl)-pyridine (Mebip) ligands. 

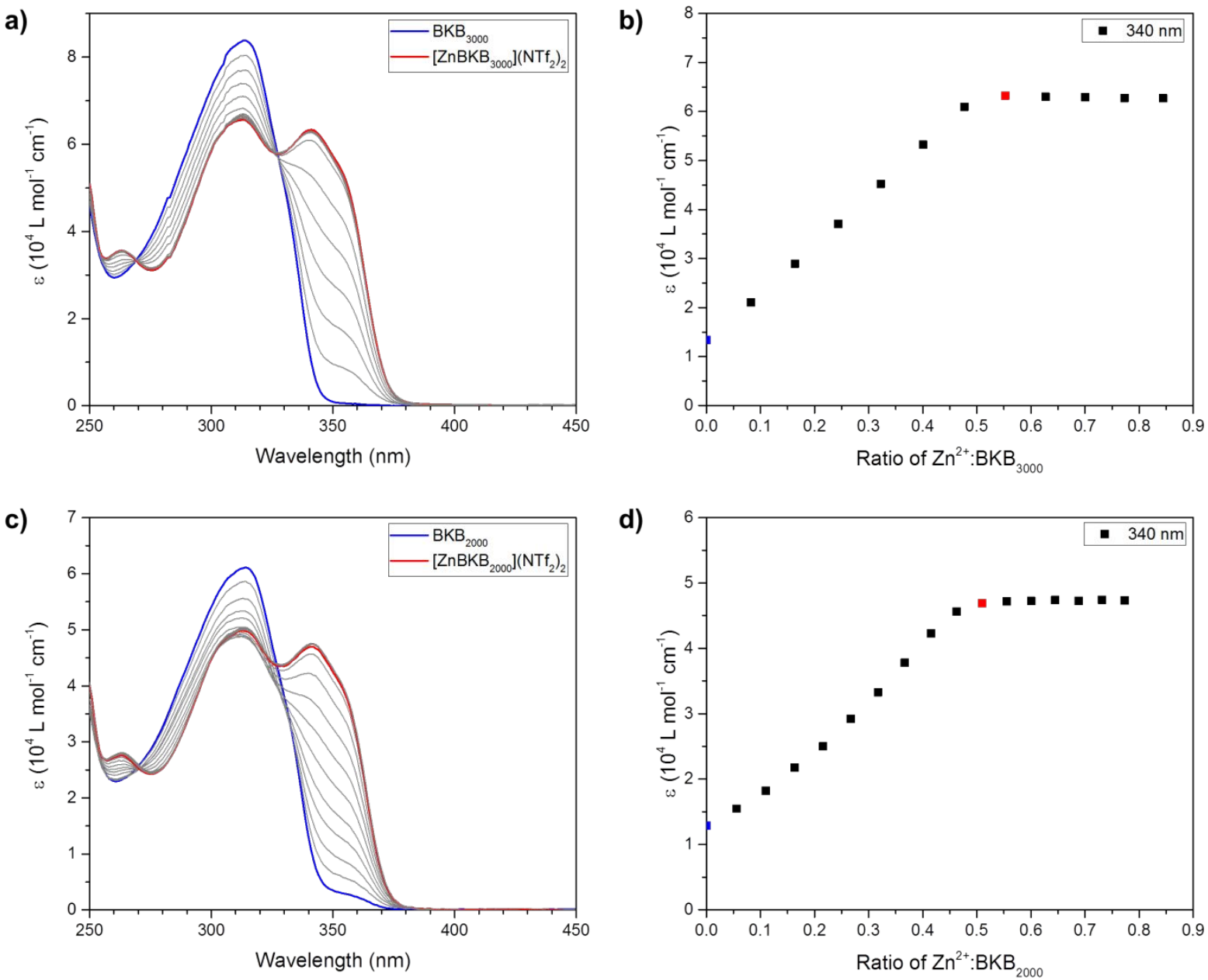

Figure S3. To demonstrate that the prepared macromonomers form complexes in the same manner as previously reported, UV-vis spectrophotometric titrations were carried out with $\mathrm{Zn}\left(\mathrm{NTf}_{2}\right)_{2}$. a) UV-vis absorption spectra of solutions of the $\mathrm{BKB}_{3000}$ macromonomer $\left(c=10 \mu \mathrm{mol} \mathrm{L}-1\right.$ in $\mathrm{CHCl}_{3} / \mathrm{MeCN}^{9: 1}$, black line). Upon titration with aliquots of $\mathrm{Zn}\left(\mathrm{NTf}_{2}\right)_{2}$ containing also the macromonomer in the same concentration $\left(c=10 \mu \mathrm{mol} \mathrm{L}^{-1}\right)$ to avoid dilution, the formation of the coordination complexes is observed. The spectrum obtained at a metal-to-ligand ratio of approximately 1:2 is highlighted (red line). b) Plot of the molar extinction coefficient at the absorption maximum of the metal-ligand complex at $340 \mathrm{~nm}$ as shown in (a) as a function of the molar ratio between added $\mathrm{Zn}\left(\mathrm{NTf}_{2}\right)_{2}$ and the $\mathbf{B K B}_{\mathbf{3 0 0 0}}$ macromonomer. These titrations confirm that the conversion to the 1:2 coordination complex occurs at a 1:2 metal-to-ligand ratio in the same way as previously reported. ${ }^{1} \mathrm{c}$ ) UV-vis absorption spectra of solutions of the $\mathbf{B K B}_{2000}$ macromonomer ( $c=10 \mu \mathrm{mol} \mathrm{L}^{-1}$ in $\mathrm{CHCl}_{3} / \mathrm{MeCN}$ 9:1, black line). The spectrum obtained at metal-to-ligand ratio of approximately $1: 2$ is highlighted (red line). d) Plot of the molar extinction coefficient at the absorption maximum of the metal-ligand complex at $340 \mathrm{~nm}$ as shown in (c) as a function of the ratio between added $\mathrm{Zn}\left(\mathrm{NTf}_{2}\right)_{2}$ and the $\mathbf{B K}_{\mathbf{2 0 0 0}}$ macromonomer. 

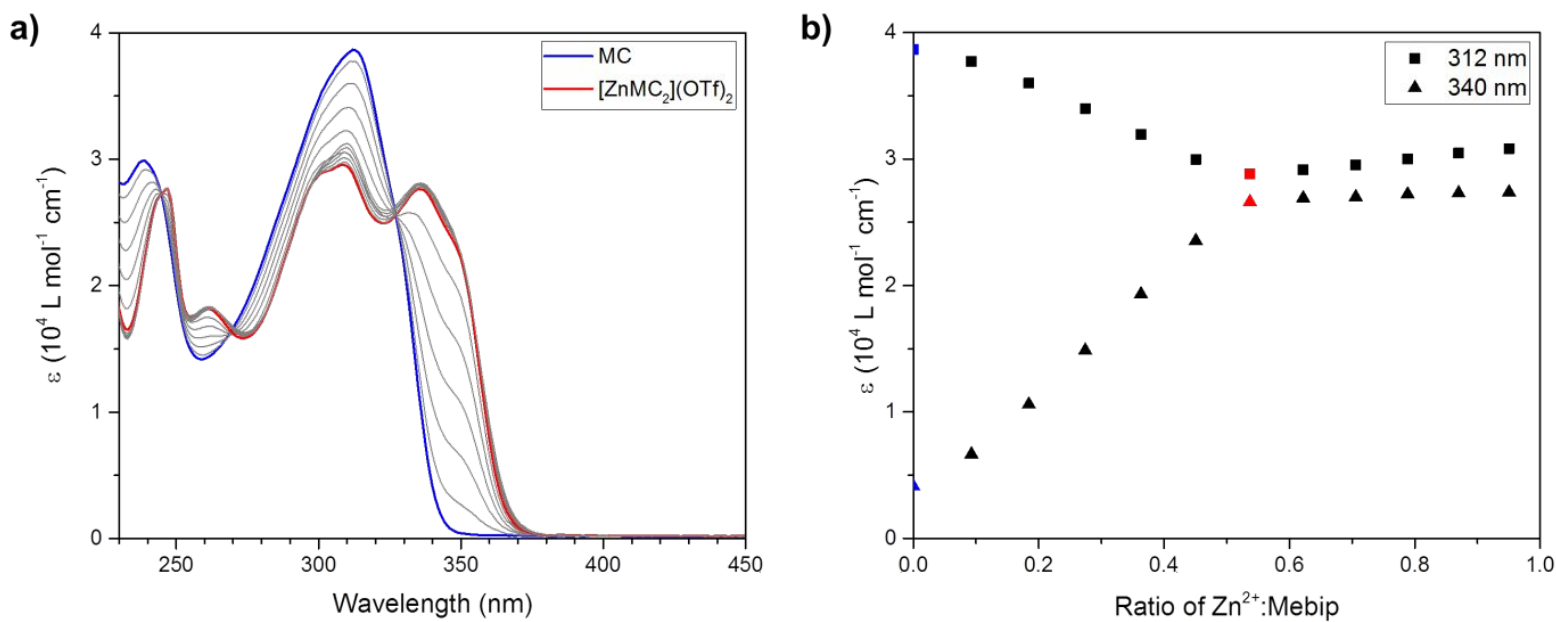

Figure S4. UV-vis spectrophotometric titrations were carried out with $\mathrm{MC}$ and $\mathrm{Zn}(\mathrm{OTf})_{2}$ to demonstrate that the complex formation occurs in the same manner as previously reported for $\mathrm{Zn}\left(\mathrm{NTf}_{2}\right)_{2} \cdot{ }^{1,2}$ a) UV-vis absorption spectra of solutions of model compound $\mathrm{MC}$ ( $c=25 \mu \mathrm{mol} \mathrm{L}-1$ in $\mathrm{MeCN}$, blue line). Upon titration with aliquots of $\mathrm{Zn}(\mathrm{OTf})_{2}$, containing also $\mathrm{MC}$ in the same concentration $\left(c=25 \mu \mathrm{mol} \mathrm{L}^{-1}\right)$ to avoid dilution, the formation of the coordination complexes is observed at a metal-to-ligand ratio of 1:2 (red line). b) Extinction of the free ligand ( $312 \mathrm{~nm}$ ) and the metal-ligand complex (340 nm) as a function of the ratio of added $\mathrm{Zn}\left(\mathrm{OTf}_{2}\right)_{2}$ and the ligand.

a)

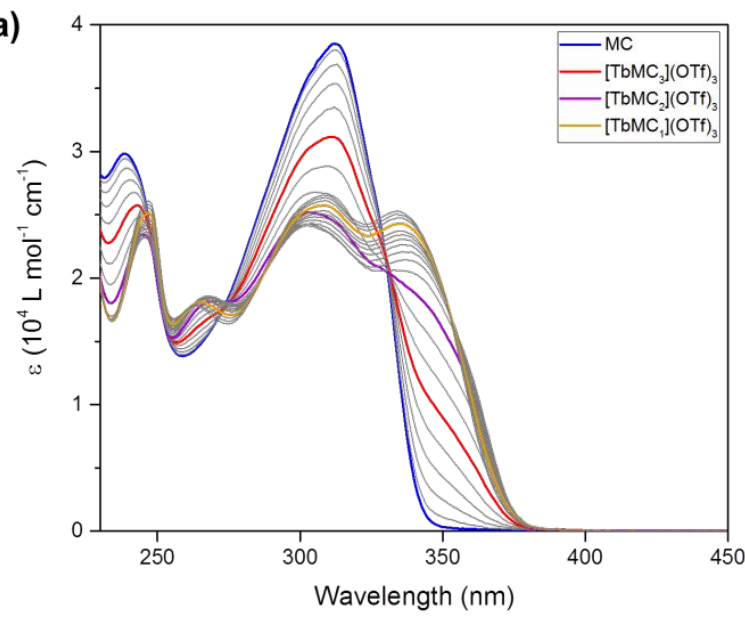

b)

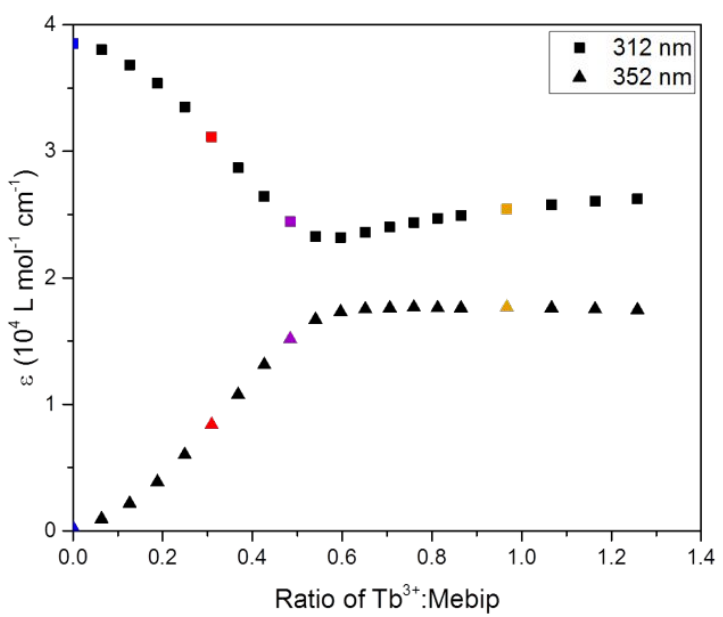

Figure S5. UV-vis spectrophotometric titrations were carried out with $\mathrm{Tb}(\mathrm{OTf})_{3}$ and the model compound $\mathbf{M C}$ to demonstrate that the complex formation occurs in the same manner as previously reported for $\left.\left.\mathrm{Tb}_{(\mathrm{ClO}}\right)_{3} \cdot{ }^{1,3,4} \mathrm{a}\right) \mathrm{UV}$ vis absorption spectra of solutions of model compound $\mathbf{M C}$ ( $c=25 \mu \mathrm{mol} \mathrm{\textrm {L } ^ { - 1 }}$ in $\mathrm{MeCN}$, blue line). Upon titration with aliquots of $\mathrm{Tb}(\mathrm{OTf})_{3}$, containing also $\mathrm{MC}$ in the same concentration $\left(c=25 \mu \mathrm{mol} \mathrm{L}^{-1}\right)$ to avoid dilution, the formation of the coordination complexes is observed. The spectra obtained at metal-to-ligand ratios of 1:3, 1:2, and 1:1 are highlighted (red, purple, and orange line, respectively). b) Extinction of the free ligand ( $312 \mathrm{~nm}$ ) and the metal-ligand complex $\left(352 \mathrm{~nm}\right.$ ) as a function of the ratio between added $\mathrm{Tb}(\mathrm{OTf})_{3}$ and the ligand. 
a)

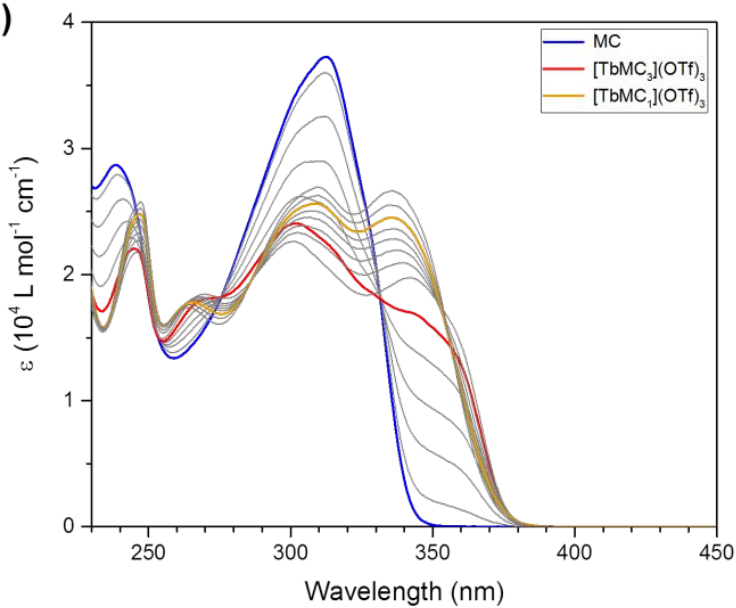

b)

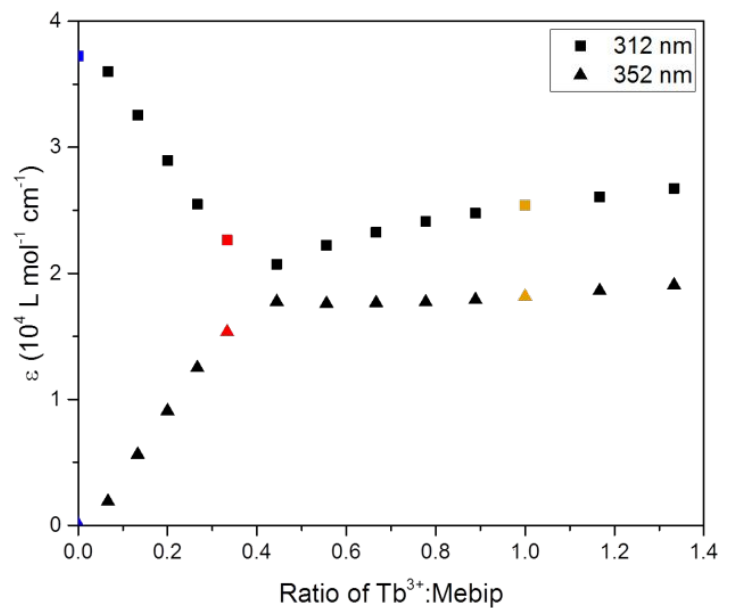

Figure S6. UV-vis spectrophotometric titrations were carried out with $\mathrm{Tb}(\mathrm{OTf})_{3}$ and the model compound $\mathrm{MC}$ at higher concentrations $\left(c=200 \mu \mathrm{mol} \mathrm{L}^{-1}\right)$ to unambiguously demonstrate the formation of 1:3 metal-to-ligand complexes upon titration with $\left.\mathrm{Tb}\left(\mathrm{ClO}_{4}\right)_{3}\right)^{1,3,4}$ a) UV-vis absorption spectra of solutions of model compound $\mathrm{MC}(\mathrm{c}=$ $200 \mu \mathrm{mol} \mathrm{L}^{-1}$ in MeCN, blue line). Upon titration with aliquots of $\mathrm{Tb}(\mathrm{OTf})_{3}$, containing also $\mathrm{MC}$ in the same concentration $\left(c=25 \mu \mathrm{mol} \mathrm{L}^{-1}\right.$ ) to avoid dilution, the formation of the coordination complexes is observed. The spectra obtained at metal-to-ligand ratios of 1:3, and 1:1 are highlighted (red and orange line, respectively). b) Absorption intensity of the free ligand $(312 \mathrm{~nm})$ and the metal-ligand complex $(352 \mathrm{~nm})$ as a function of the ratio between added $\mathrm{Tb}(\mathrm{OTf})_{3}$ and the ligand. 


\subsection{Thermal Properties S7-S15}

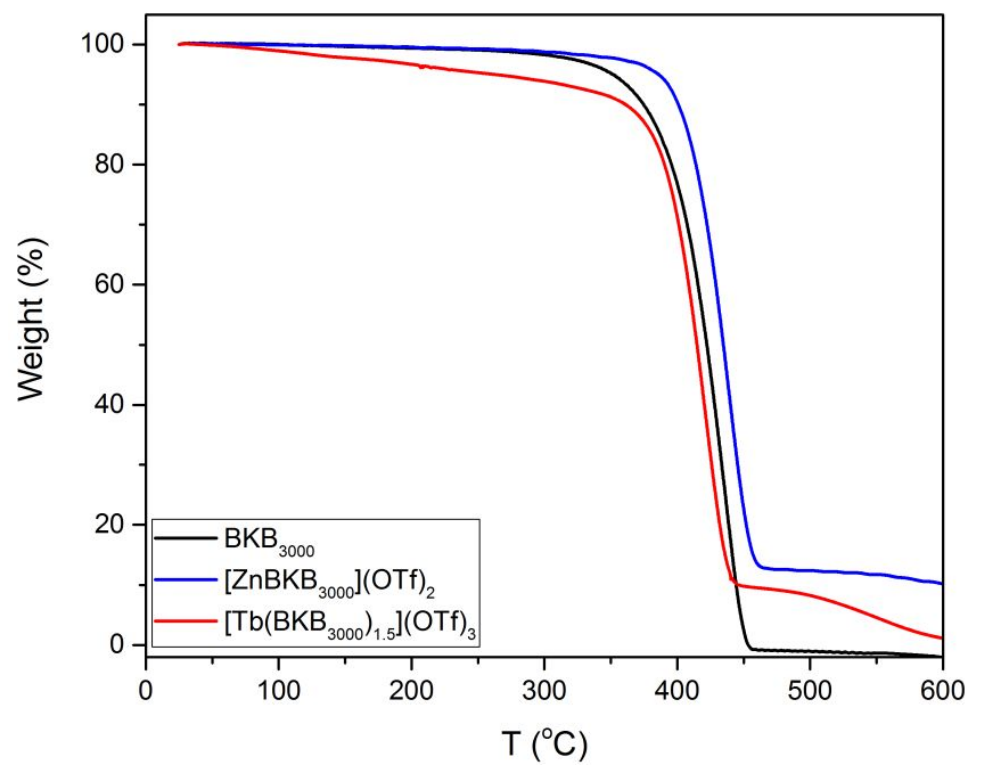

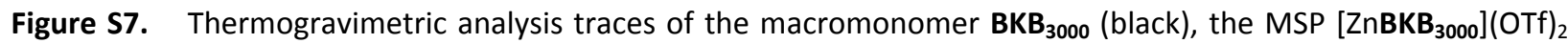
(blue), and the MSP $\left[\mathrm{Tb}\left(\mathrm{BKB}_{3000}\right)_{1.5}\right](\mathrm{OTf})_{3}$ (red) recorded in air. The traces reveal a $5 \%$ weight loss at temperatures of $351{ }^{\circ} \mathrm{C}$ for $\mathrm{BKB}_{3000}, 386^{\circ} \mathrm{C}$ for $\left[\mathrm{ZnBKB}{ }_{3000}\right](\mathrm{OTf})_{2}$, and $263^{\circ} \mathrm{C}$ in the case of $\left[\mathrm{Tb}\left(\mathrm{BKB}_{3000}\right)_{1.5}\right](\mathrm{OTf})_{3}$. 


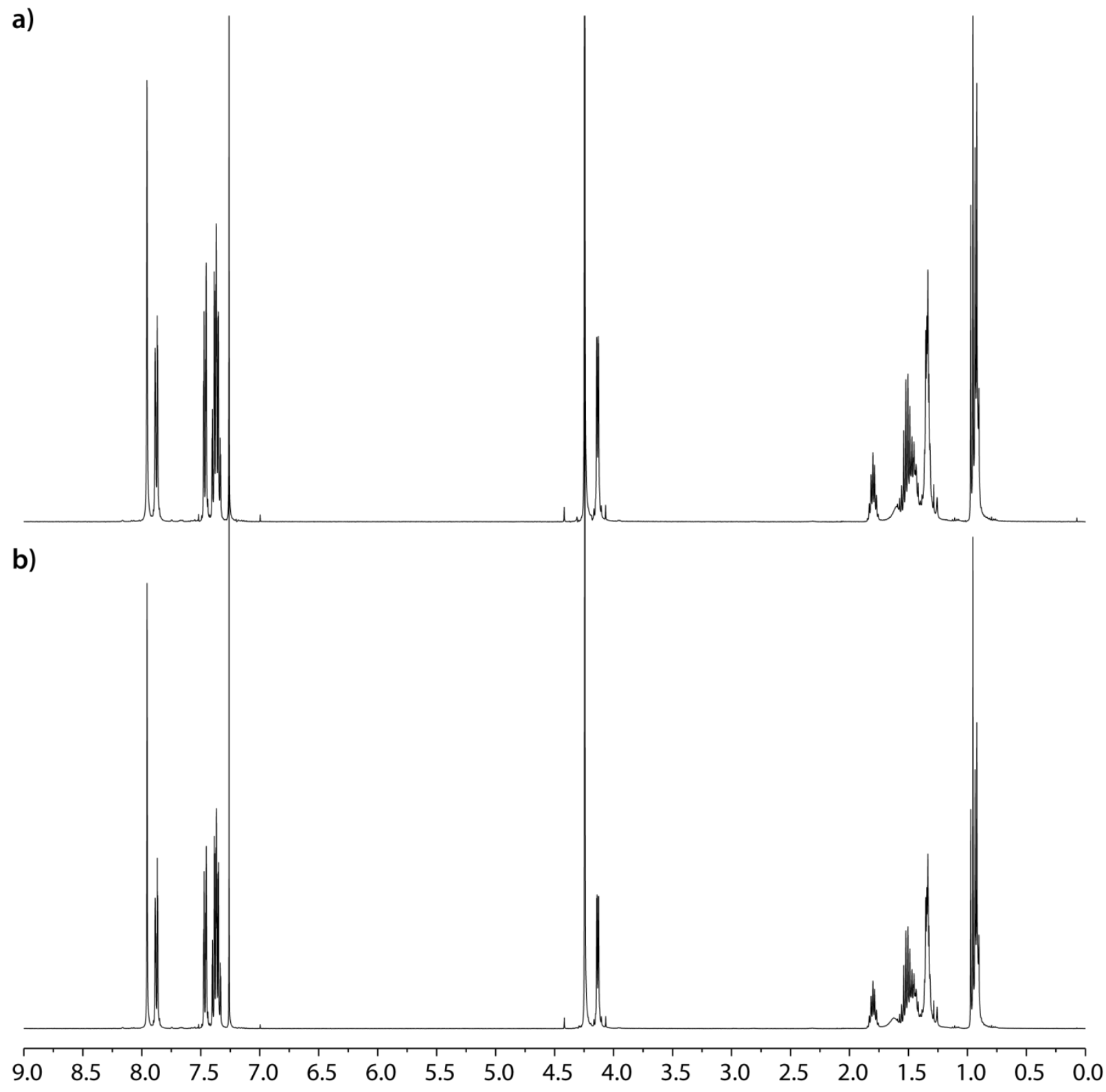

Figure S8. Comparison of the ${ }^{1} \mathrm{H}$ NMR spectra $\left(\mathrm{CDCl}_{3}, 400 \mathrm{MHz}\right)$ of the model compound $\mathrm{MC}(\mathrm{a})$ before and (b) after keeping a sample on a hot plate for $10 \mathrm{~min}$ at a temperature of $250^{\circ} \mathrm{C}$. The spectra appear identical, suggesting that no changes to the chemical structure occur when maintaining samples at this temperature. 

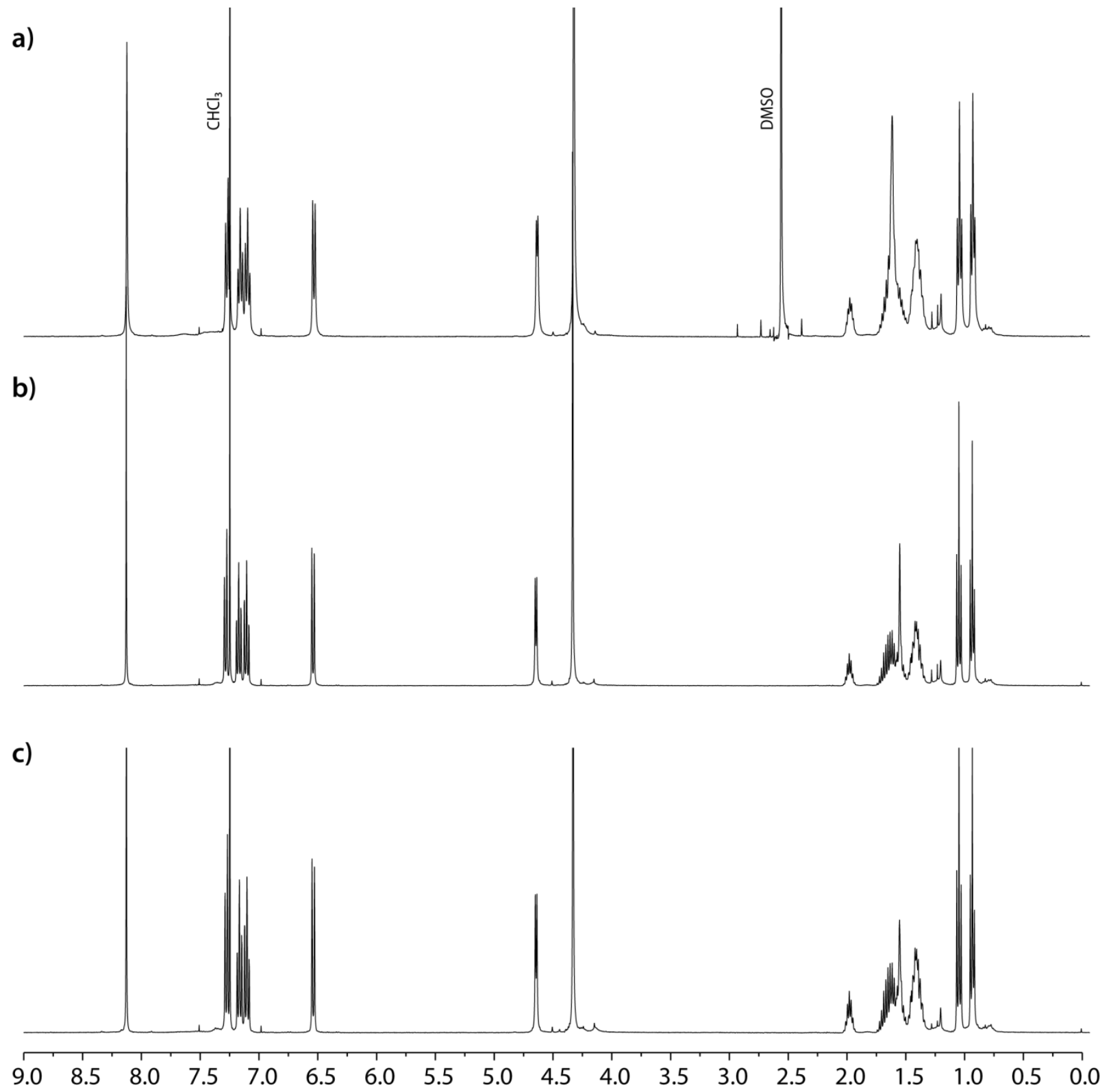

Figure 59. Comparison of the ${ }^{1} \mathrm{H}$ NMR spectra $\left(\mathrm{CDCl}_{3}, 400 \mathrm{MHz}\right)$ of the $\left[\mathrm{ZnMC}_{2}\right](\mathrm{OTf})_{2}$ complex (a) before and after heating samples on a hot plate (b) for $10 \mathrm{~min}$ at $200^{\circ} \mathrm{C}$ as well as (c) for $10 \mathrm{~min}$ at $250^{\circ} \mathrm{C}$. The spectra do not indicate changes, suggesting that the metal-ligand complexes retain their structural integrity when heating up to temperatures of $250^{\circ} \mathrm{C}$. 

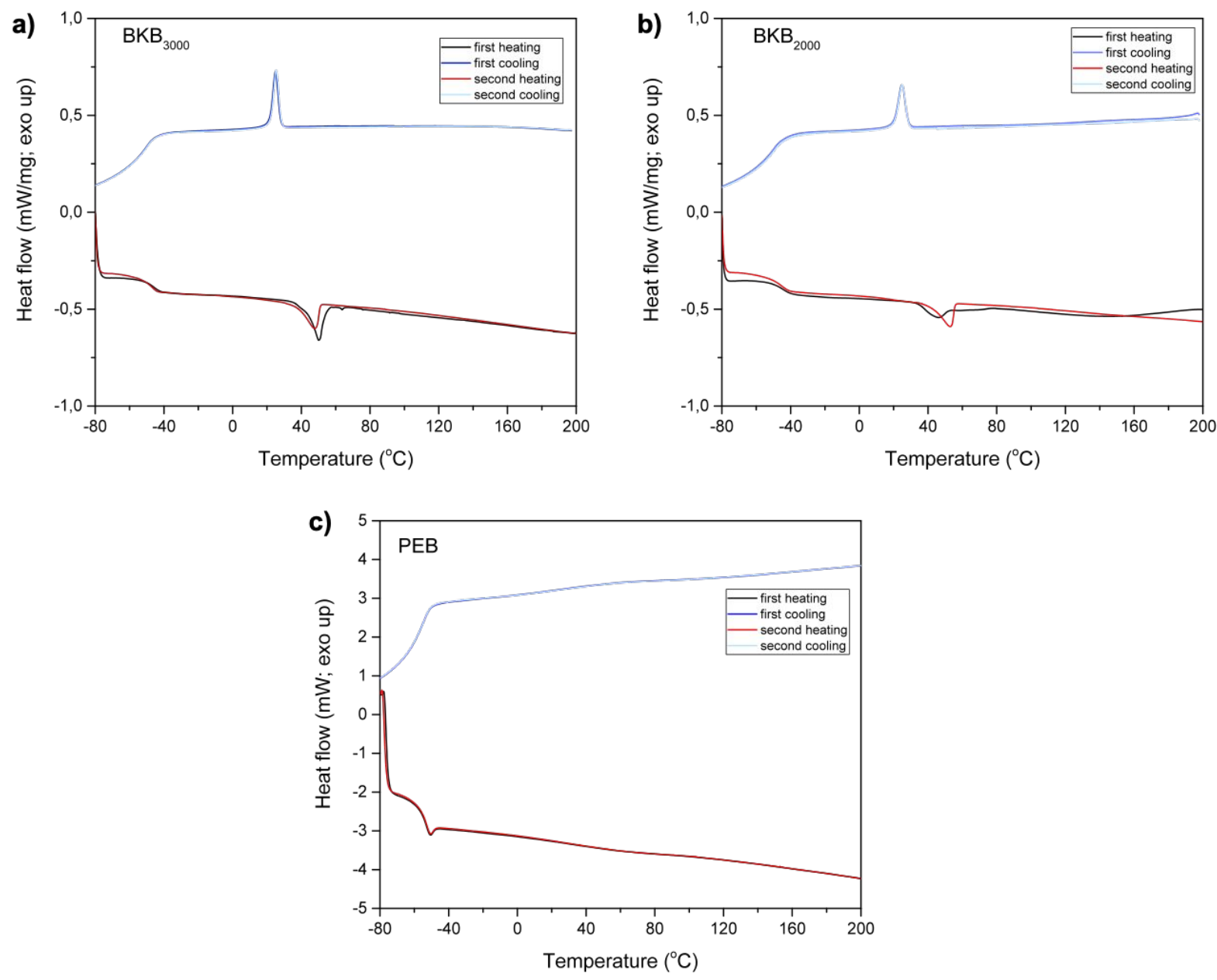

Figure S10. Differential scanning calorimetry (DSC) traces of the macromonomers $\mathbf{B K B}_{3000}\left(M_{\mathrm{n}}=3800 \mathrm{~g} \mathrm{~mol}^{-1}\right)$, BKB $_{2000}\left(M_{\mathrm{n}}=3000 \mathrm{~g} \mathrm{~mol}^{-1}\right)$, and of the poly(ethylene-co-butylene) (PEB; Krasol ${ }^{\circledR}, 2100 \mathrm{~g} \mathrm{~mol}^{-1}$ ) core. a) First and second heating and cooling traces of compression molded samples of $\mathbf{B K B}_{3000}$. b) First and second heating and cooling traces of compression molded samples of $\mathbf{B K B}_{2000}$. c) First and second heating and cooling traces of samples of poly(ethylene-co-butylene). The experiments were conducted at a heating rate of $10^{\circ} \mathrm{C} \mathrm{min}^{-1}$ in a $\mathrm{N}_{2}$ atmosphere. 
a)

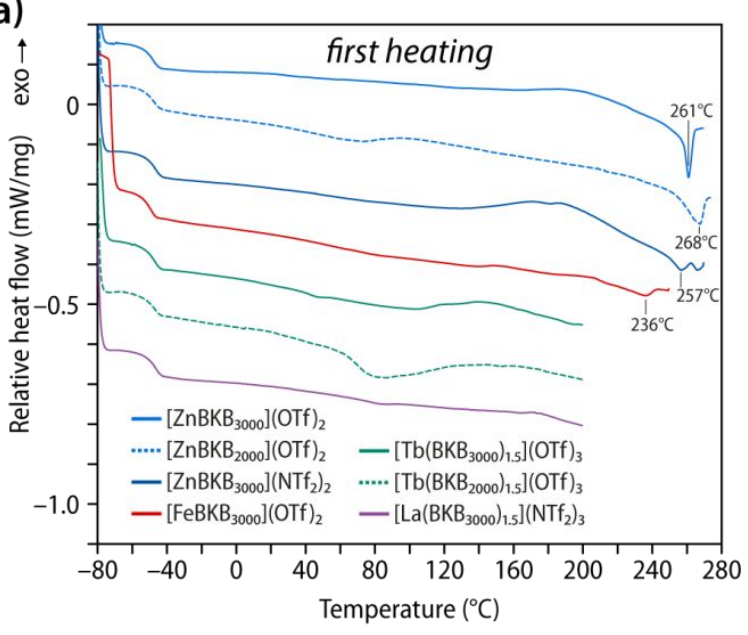

b)

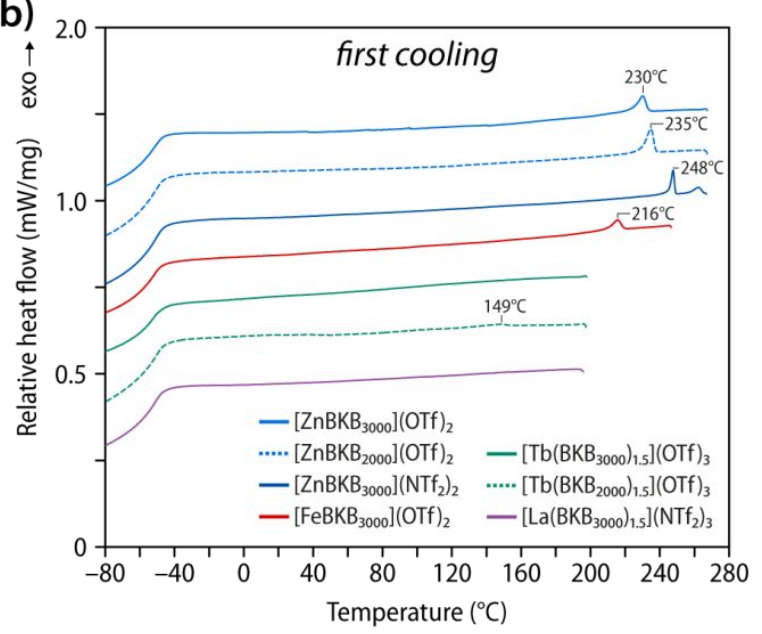

Figure S11. Comparison of the differential scanning calorimetry (DSC) traces of the macromonomers and metallosupramolecular polymers. a) First heating and (b) first cooling scans of compression molded samples of $\left[\mathrm{ZnBKB}_{3000}\right](\mathrm{OTf})_{2}, \quad\left[\mathrm{ZnBKB}_{2000}\right](\mathrm{OTf})_{2}, \quad\left[\mathrm{ZnBKB}_{3000}\right]\left(\mathrm{NTf}_{2}\right)_{2}, \quad\left[\mathrm{ZnBKB}_{3000}\right](\mathrm{OTf})_{2}, \quad\left[\mathrm{~Tb}\left(\mathrm{BKB}_{3000}\right)_{1.5}\right](\mathrm{OTf})_{3}$, $\left[\mathrm{Tb}\left(\mathrm{BKB}_{2000}\right)_{1.5}\right](\mathrm{OTf})_{3}$, and $\left[\mathrm{La}\left(\mathrm{BKB}_{3000}\right)_{1.5}\right]\left(\mathrm{NTf}_{2}\right)_{3}$. The experiments were conducted at a heating rate of $10^{\circ} \mathrm{C} \mathrm{min}^{-1} \mathrm{in}$ a $\mathrm{N}_{2}$ atmosphere and the traces are vertically shifted for clarity. Measurements with lanthanoid MSPs were carried out up to $200^{\circ} \mathrm{C}$ since sample failure and loss of ordering occurs at lower temperatures in DMA and SAXS measurements.

a)

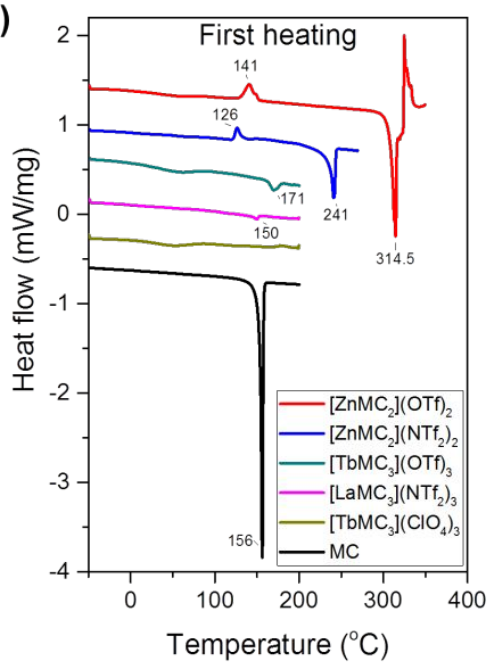

b)

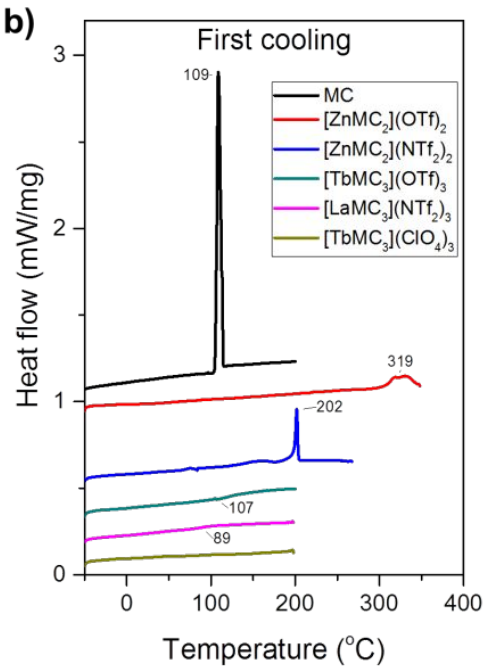

c)

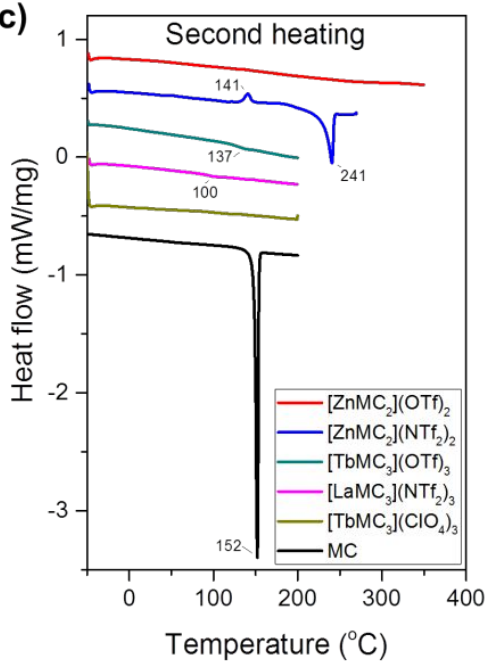

Figure S12. Comparison of the differential scanning calorimetry (DSC) traces of the model compound (MC) and its complexes with different metal salts. a) First heating, b) first cooling, and (c) second heating scans of the model compound MC, $\left[\mathrm{ZnMC}_{2}\right](\mathrm{OTf})_{2},\left[\mathrm{ZnMC}_{2}\right]\left(\mathrm{NTf}_{2}\right)_{2},\left[\mathrm{TbMC}_{3}\right](\mathrm{OTf})_{3}$, $\left[\mathrm{LaMC}_{3}\right]\left(\mathrm{NTf}_{2}\right)_{3}$, and $\left[\mathrm{TbMC}_{3}\right]\left(\mathrm{ClO}_{4}\right)_{3}$. The experiments were conducted at a heating rate of $10^{\circ} \mathrm{C} \mathrm{min}^{-1}$ in a $\mathrm{N}_{2}$ atmosphere and the traces are vertically shifted for clarity. 
a) $\left[\mathrm{ZnMC}_{2}\right](\mathrm{OTf})_{2}$

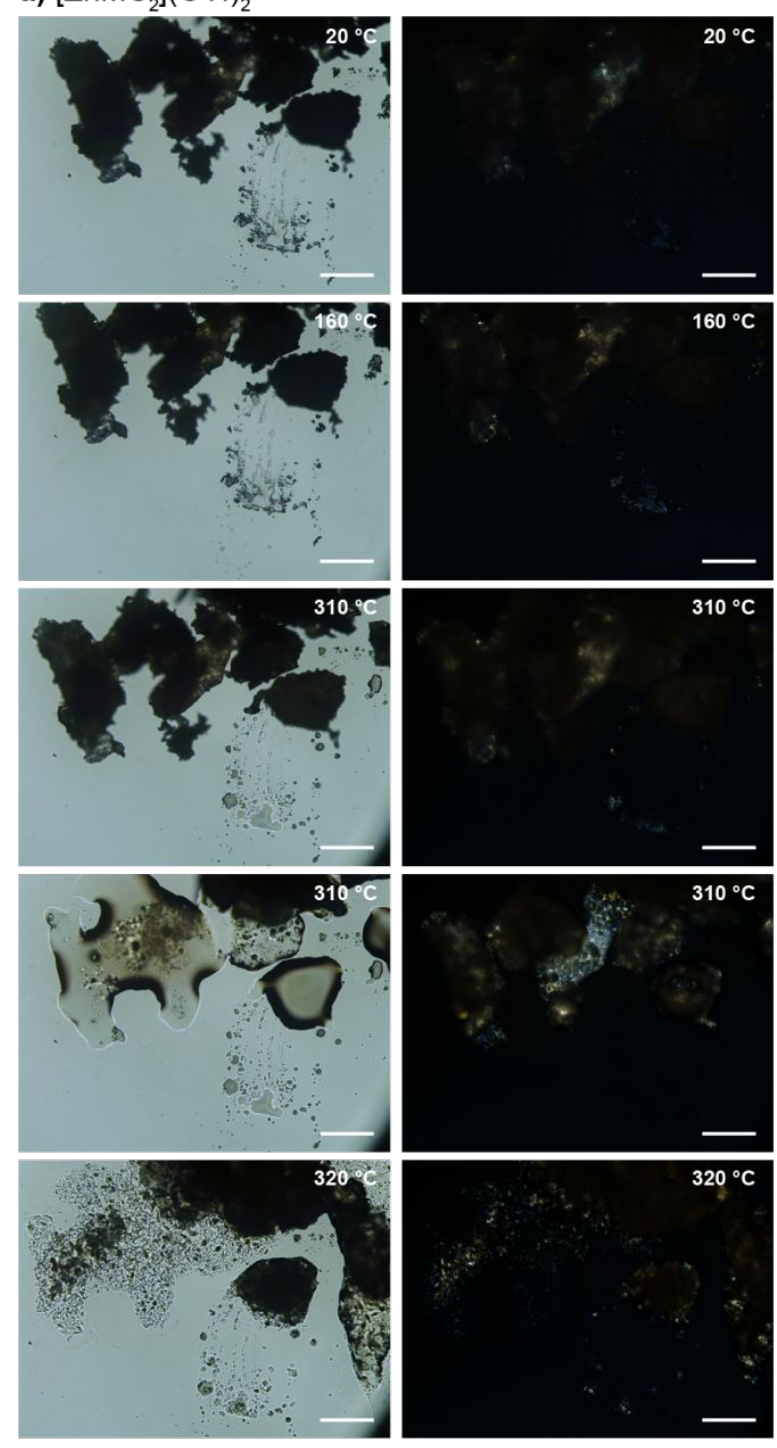

b) $\left[\mathrm{ZnMC}_{2}\right]\left(\mathrm{NTf}_{2}\right)_{2}$

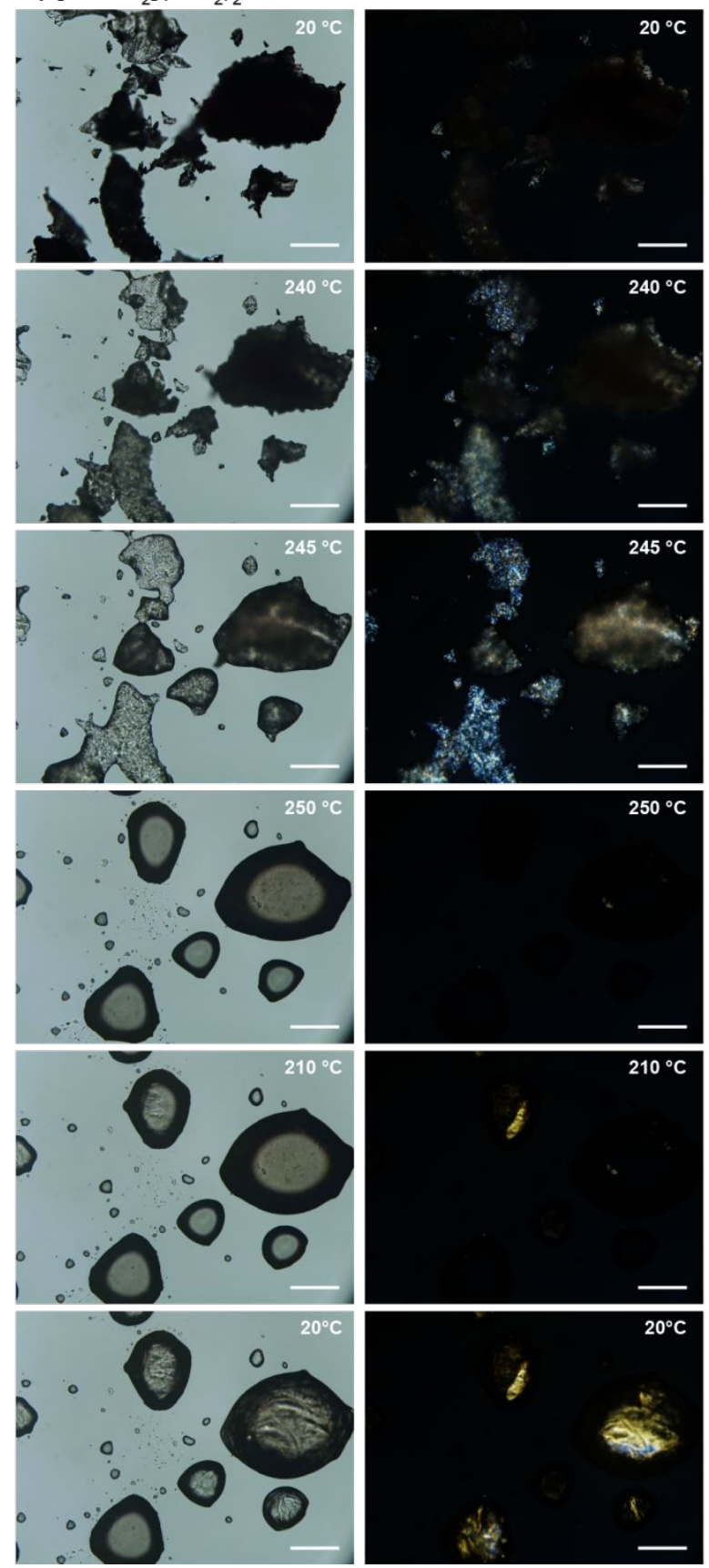

Figure S13. Optical microscopy (left) and polarized optical microscopy (right) images recorded during heating of (a) solid samples of $\left[\mathrm{ZnMC}_{2}\right](\mathrm{OTf})_{2}$ and (b) of solid samples of $\left[\mathrm{ZnMC}_{2}\right]\left(\mathrm{NTf}_{2}\right)_{2}$ during heating and cooling. The scale bars represent $100 \mu \mathrm{m}$. 
a) $\left[\mathrm{TbMC}_{3}\right](\mathrm{OTf})_{3}$
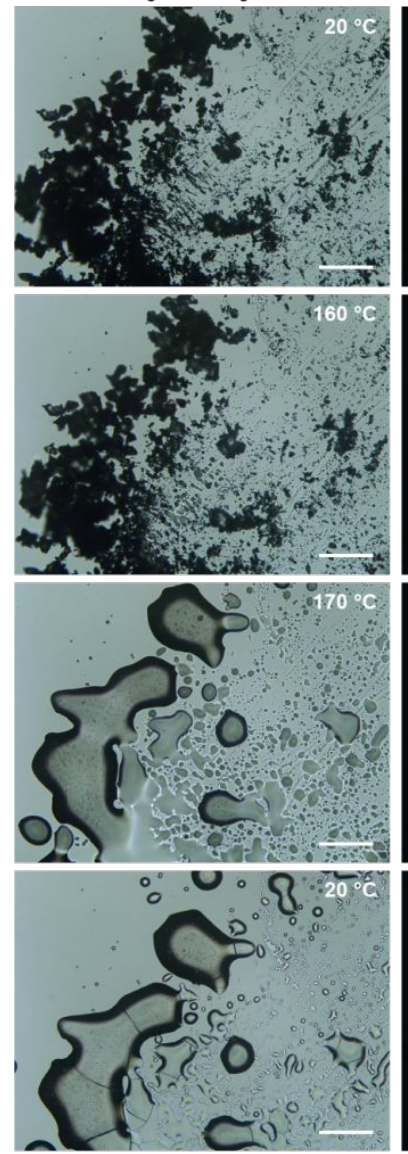
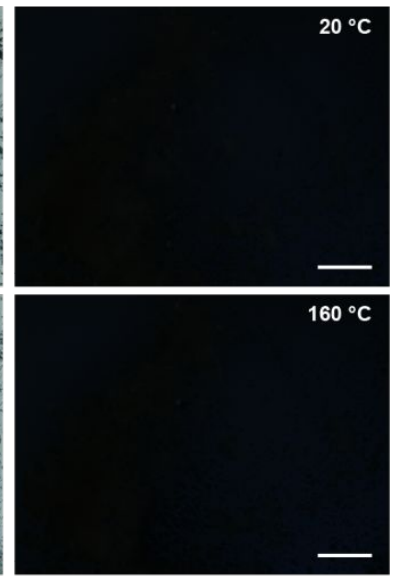

$170^{\circ} \mathrm{C}$

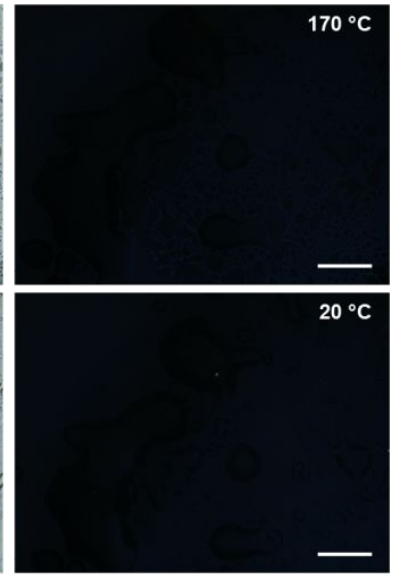

b) $\left[\mathrm{LaMC}_{3}\right]\left(\mathrm{NTf}_{2}\right)_{3}$
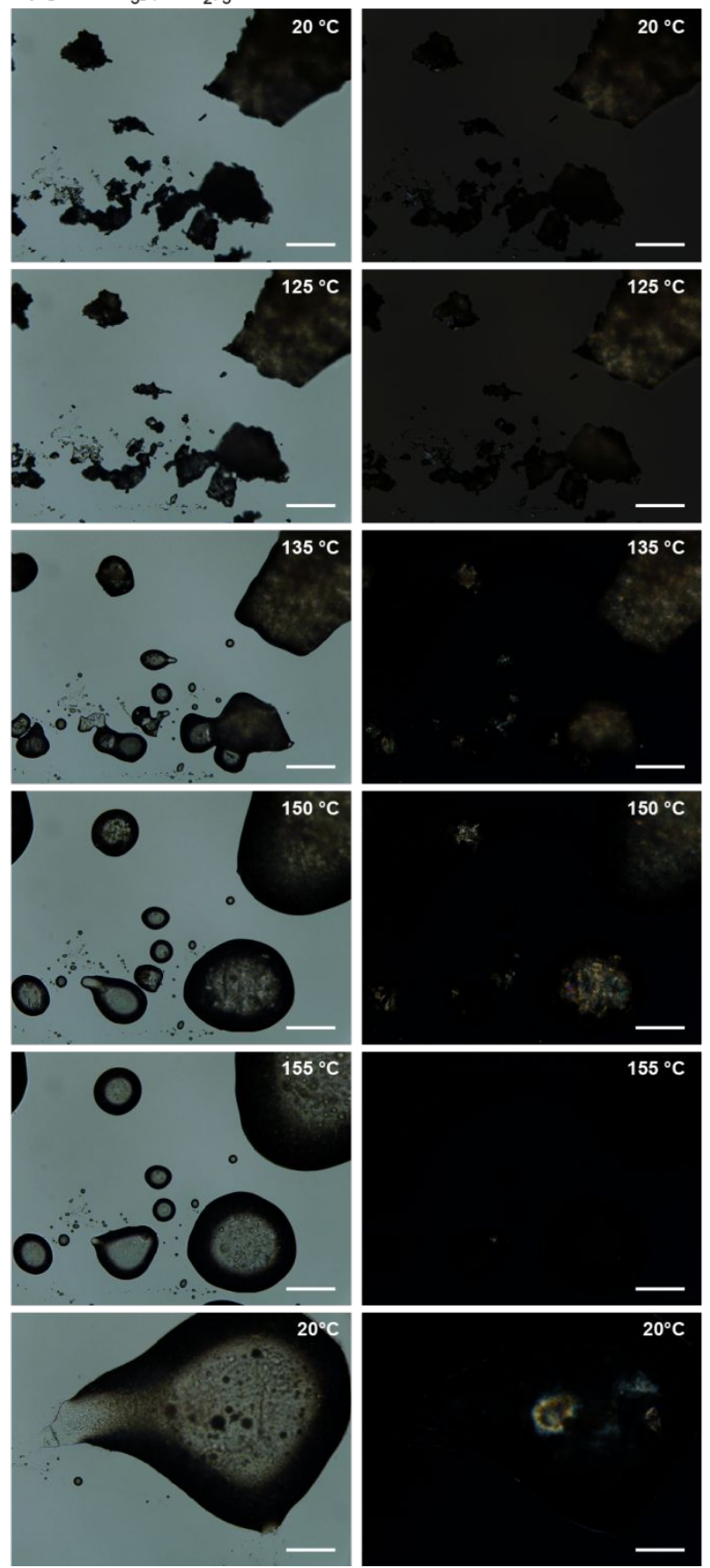

Figure S14. Optical microscopy (left) and polarized optical microscopy (right) images of solid samples of (a) $\left[\mathrm{TbMC}_{3}\right](\mathrm{OTf})_{3}$ during heating as well as (b) $\left[\mathrm{LaMC}_{3}\right]\left(\mathrm{NTf}_{2}\right)_{3}$ during heating and cooling. The scale bars represent $100 \mu \mathrm{m}$. 


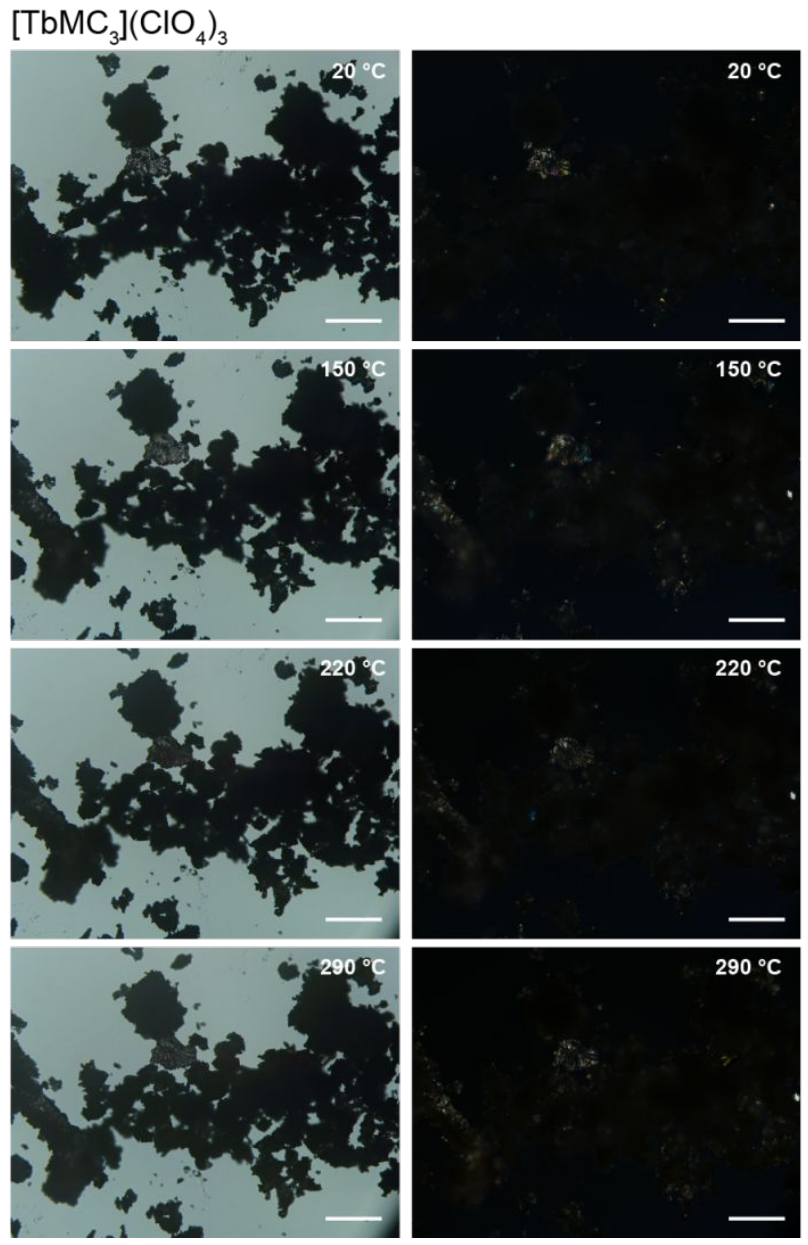

Figure S15. Optical microscopy (left) and polarized optical microscopy (right) images of solid samples of $\left[\mathrm{TbMC}_{3}\right]\left(\mathrm{ClO}_{4}\right)_{3}$ during heating. The scale bars represent $100 \mu \mathrm{m}$. 


\subsection{Small- and Wide-Angle X-ray Scattering S16-S19}

a)

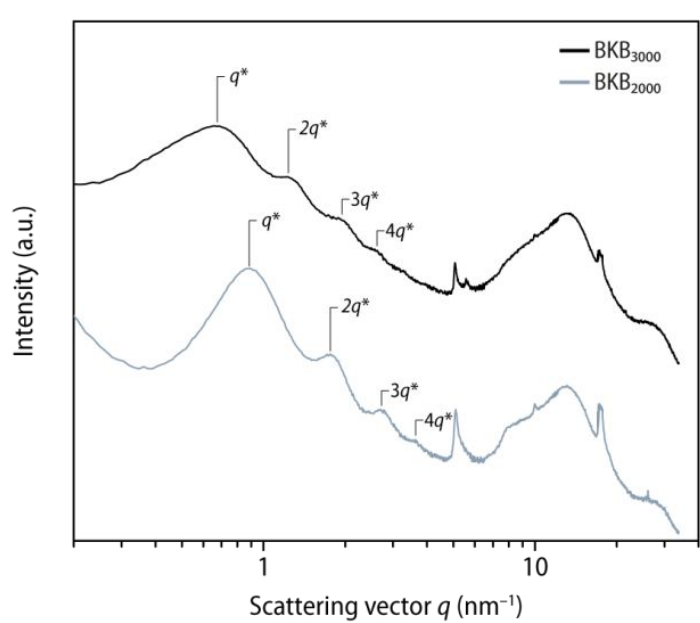

b)

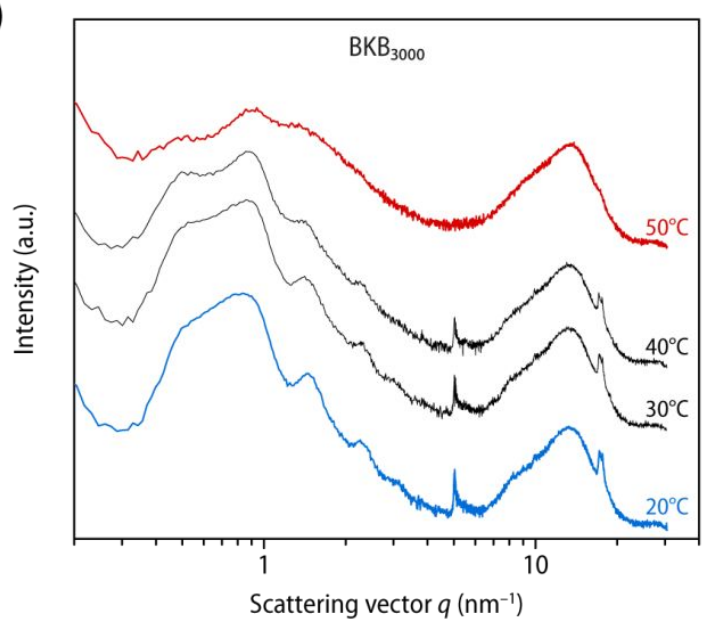

Figure S16. a) Comparison of the small- and wide-angle X-ray scattering (SAXS/WAXS) profiles of samples of the macromonomers $\mathbf{B K}_{\mathbf{3 0 0 0}}$ and $\mathbf{B K}_{\mathbf{2 0 0 0}}$. A main Bragg peak $\left(q^{*}\right)$ and integer higher-order reflections are observed that imply a lamellar morphology. b) Comparison of SAXS/WAXS profiles of a sample of $\mathbf{B K}_{\mathbf{3}} \mathbf{3}_{\mathbf{3} 00}$ upon heating to different temperatures between 20 and $50{ }^{\circ} \mathrm{C}$. A shoulder at a smaller scattering vectors is discernible. The heating and cooling rate for these temperature-dependent scattering experiments was $10{ }^{\circ} \mathrm{C} \mathrm{min}-1$ and samples were equilibrated at each temperature for $10 \mathrm{~min}$ before data collection was started. Scattering profiles are shifted vertically for clarity.

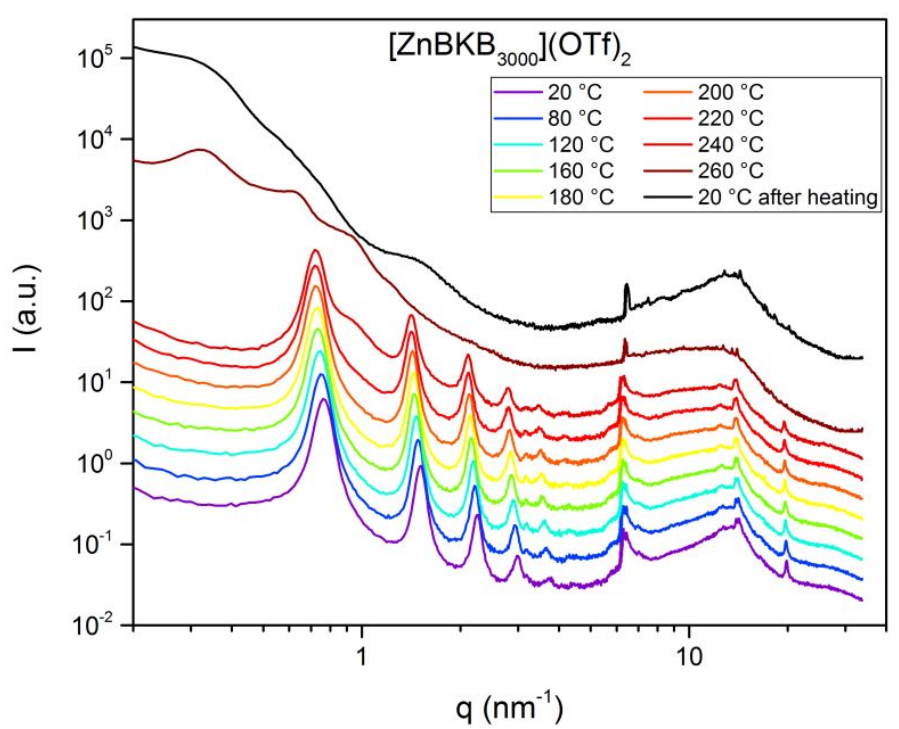

Figure S17. Comparison of the small- and wide-angle X-ray scattering (SAXS/WAXS) profiles of samples of the MSP $\left[\mathrm{ZnBKB}_{3000}\right](\mathrm{OTf})_{2}$ upon heating to different temperatures between 20 and $240{ }^{\circ} \mathrm{C}$. The heating and cooling rate for these temperature-dependent scattering experiments was $10{ }^{\circ} \mathrm{C} \mathrm{min}-1$ and samples were equilibrated at each temperature for $10 \mathrm{~min}$ before data collection was started. Scattering profiles are shifted vertically for clarity. 


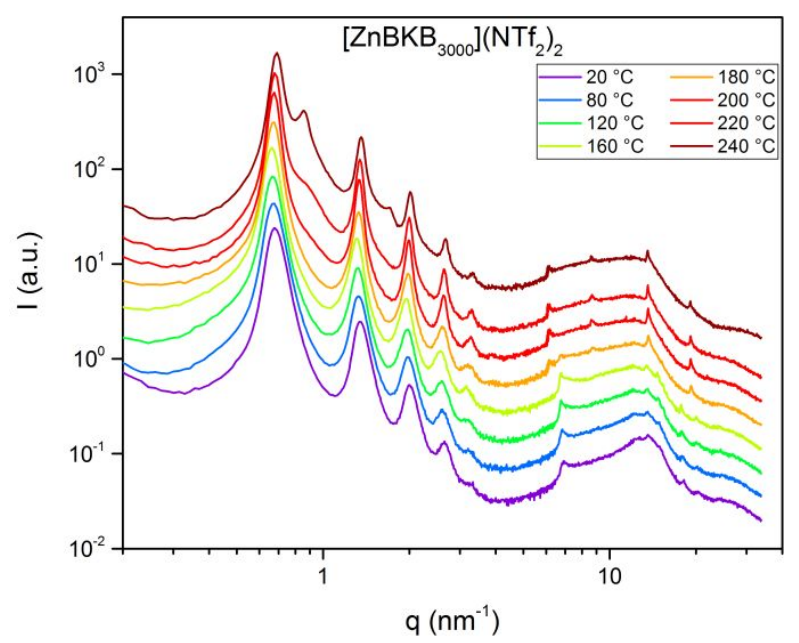

Figure S18. Comparison of the small- and wide-angle X-ray scattering (SAXS/WAXS) profiles of samples of the MSP $\left[\mathrm{ZnBKB}_{3000}\right]\left(\mathrm{NTf}_{2}\right)_{2}$ upon heating to different temperatures between 20 and $240^{\circ} \mathrm{C}$. The heating and cooling rate for these temperature-dependent scattering experiments was $10^{\circ} \mathrm{C} \mathrm{min}^{-1}$ and samples were equilibrated at each temperature for $10 \mathrm{~min}$ before data collection was started. Scattering profiles are shifted vertically for clarity.

a)

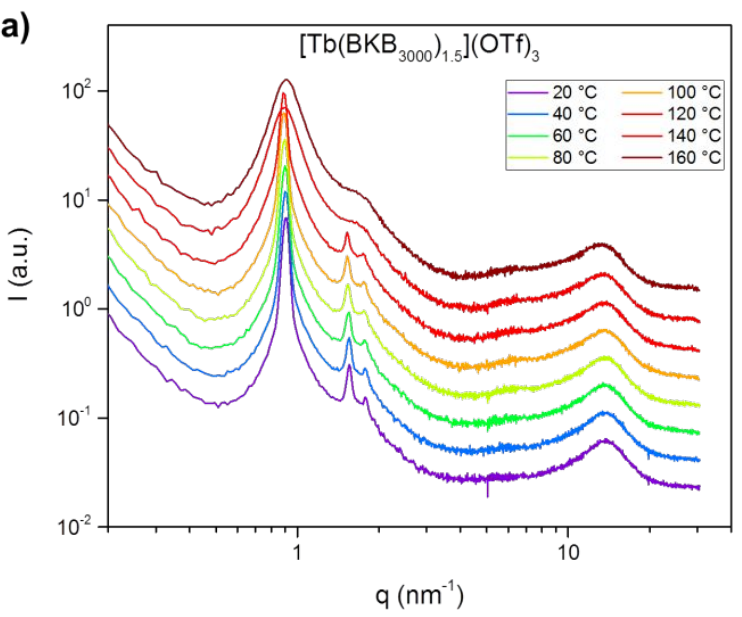

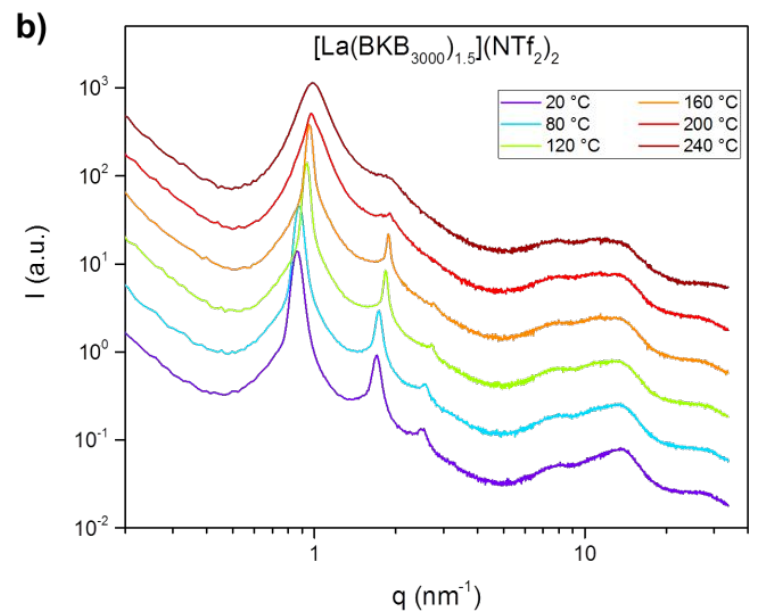

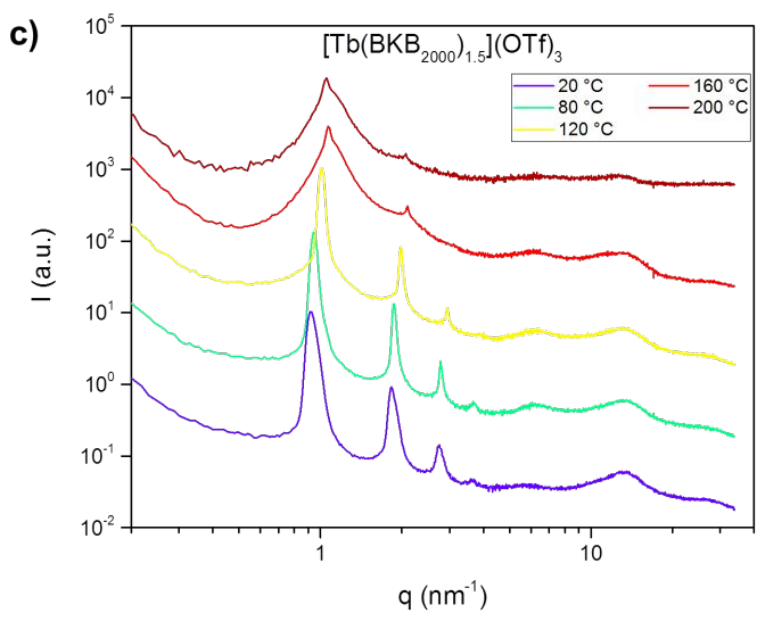

Figure S19. Comparison of the small- and wide-angle X-ray scattering (SAXS/WAXS) profiles of samples of the MSPs (a) $\left[\mathrm{Tb}\left(\mathrm{BKB}_{3000}\right)_{1.5}\right](\mathrm{OTf})_{3}$, b) $\left[\mathrm{La}\left(\mathrm{BKB}_{3000}\right)_{1.5}\right]\left(\mathrm{NTf}_{2}\right)_{3}$, and (c) $\left[\mathrm{Tb}\left(\mathbf{B K B}_{2000}\right)_{1.5}\right](\mathrm{OTf})_{3}$ upon heating to different temperatures between 20 and 160,240 , and $200^{\circ} \mathrm{C}$, respectively. The heating and cooling rate for these temperature-dependent scattering experiments was $10^{\circ} \mathrm{C} \mathrm{min}-1$ and samples were equilibrated at each temperature for $10 \mathrm{~min}$ before data collection was started. Scattering profiles are shifted vertically for clarity. 


\subsection{Thermomechanical Characterization $\mathbf{S 2 0}$}

a)

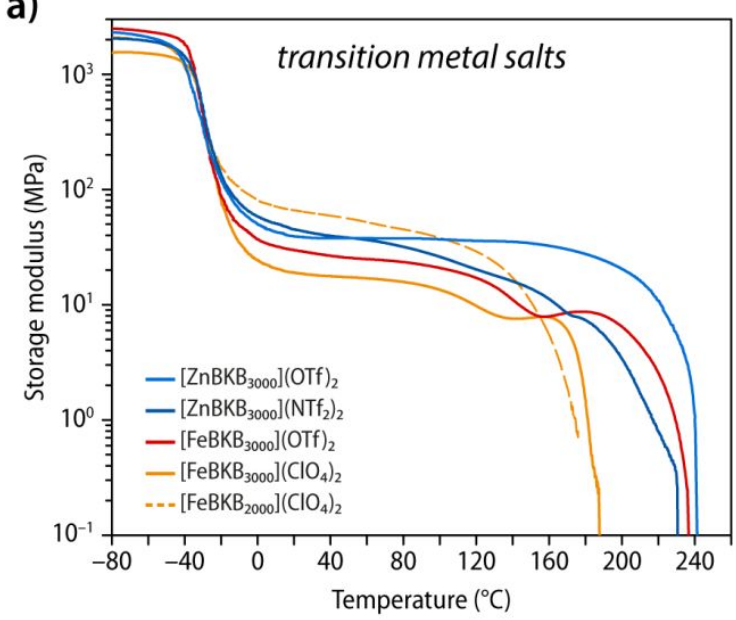

b)

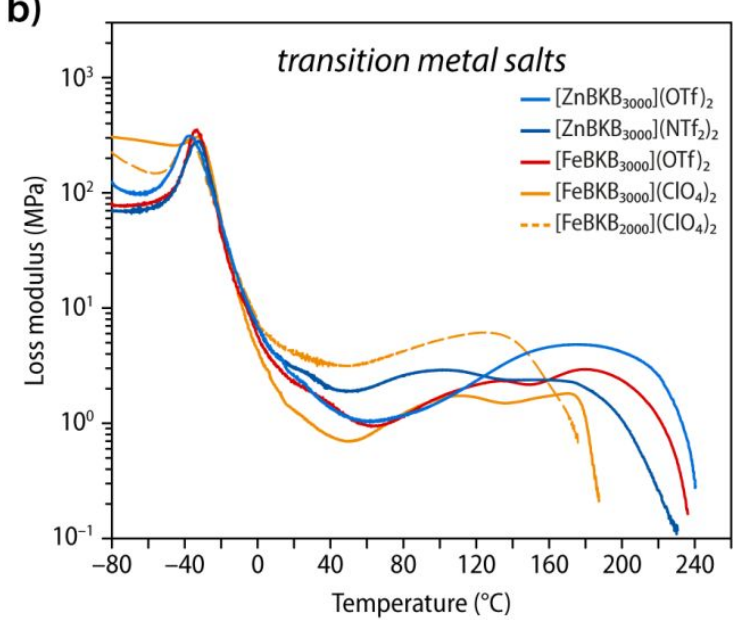

c)

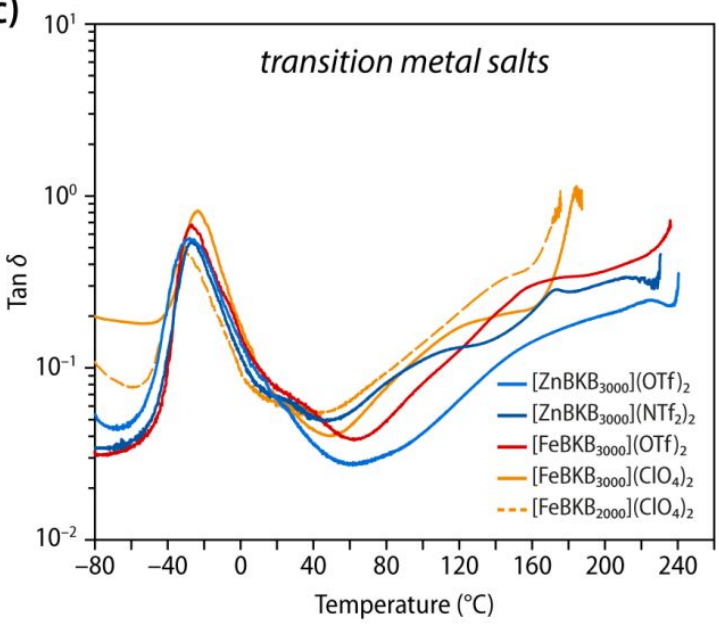

d)

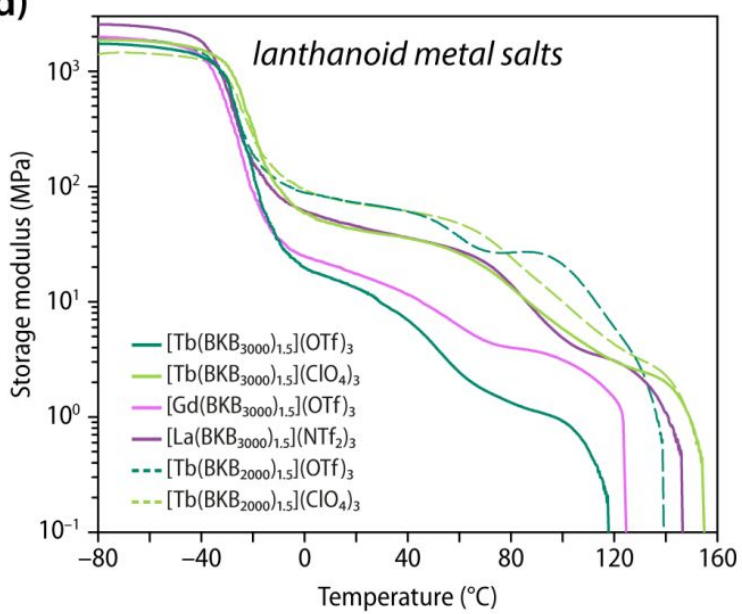

e)

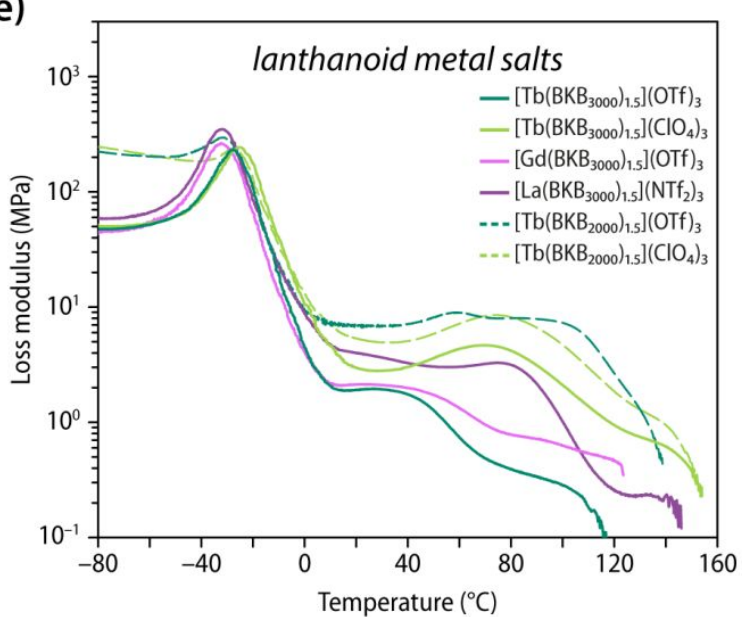

f)

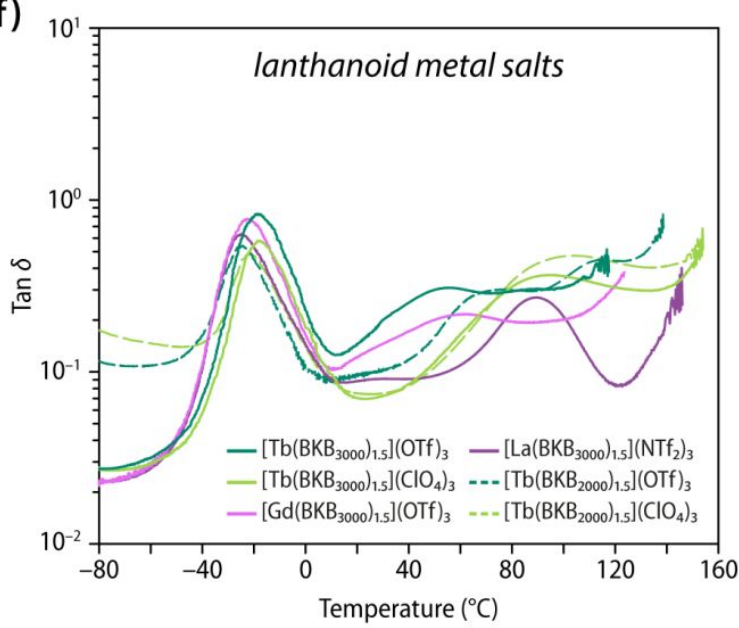

Figure S20. Comparison of the storage moduli, loss moduli, and $\tan \delta$ traces determined by dynamic mechanical analysis (DMA). a) Storage moduli, b) loss moduli, and (c) $\tan \delta$ traces of MSPs with different transition metal ions and varying counter ions and polymer cores. d) Storage moduli, e) loss moduli, (f) $\tan \delta$ traces of MSPs with different lanthanide metal ions with varying counter ions and polymer cores. 


\subsection{Rheological Investigations S21-S36}
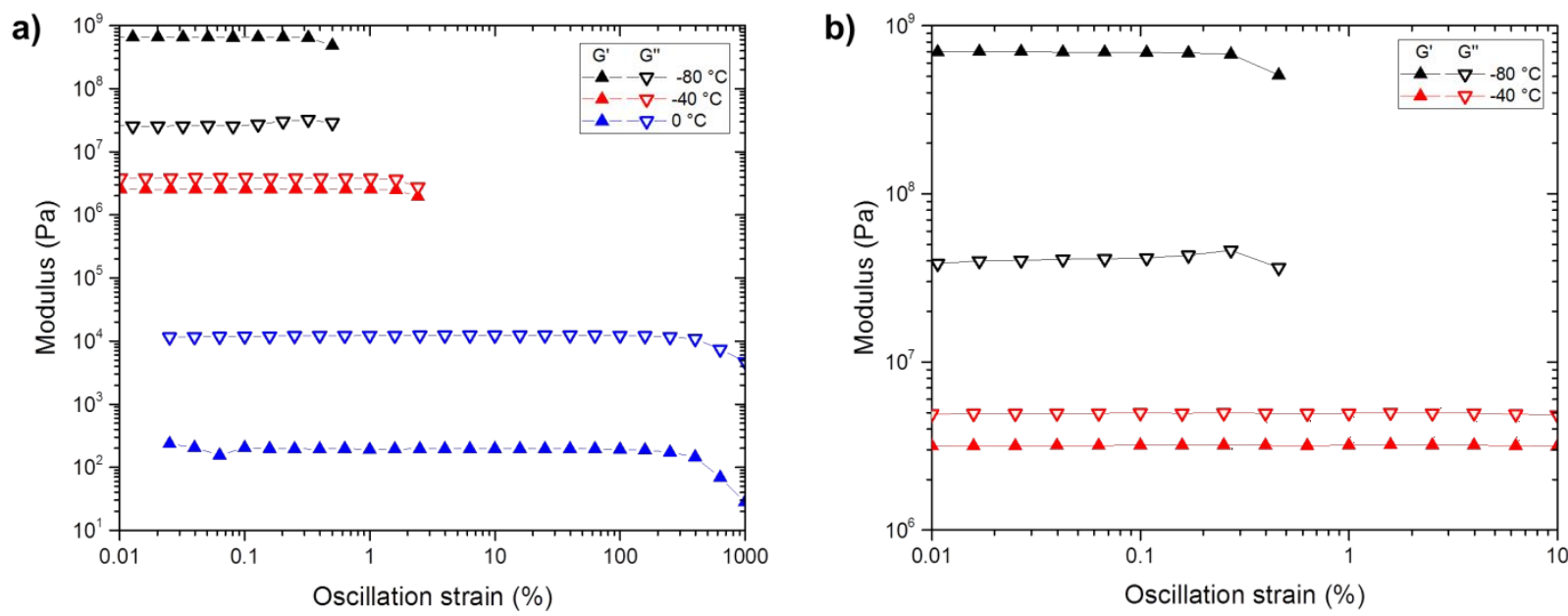

Figure S21. Plots of the modulus against the oscillation strain as determined by strain sweep experiments with poly(ethylene-co-butylene)s at a fixed angular frequency of $10 \%$. The measurements were employed to determine the limit of the linear response behavior of a) PEB 3000 and b) PEB 2000 .
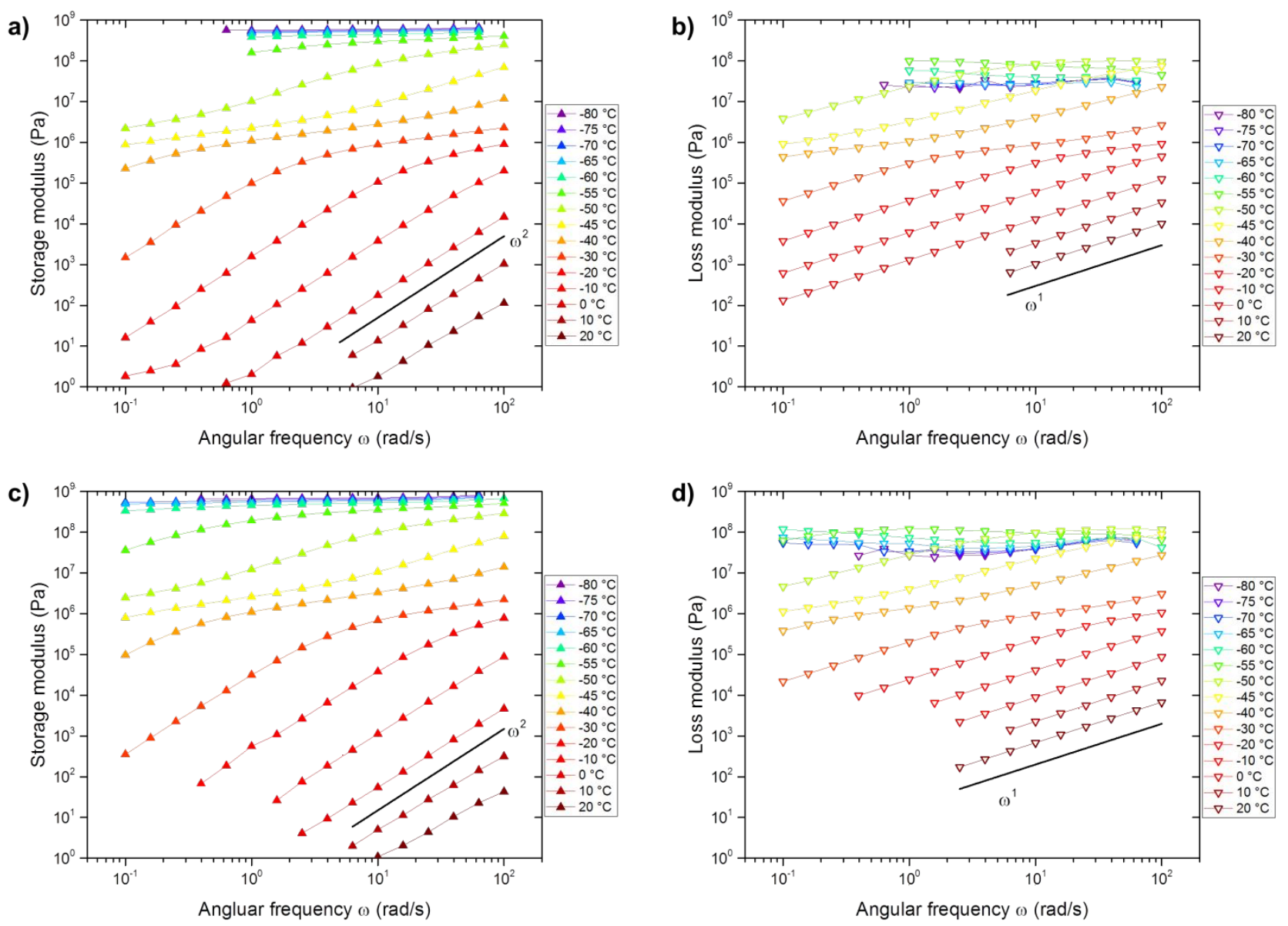

Figure S22. Linear shear rheology of poly(ethylene-co-butylene)s. Storage and loss moduli of (a,b) PEB 3000 and (c,d) $\mathrm{PEB}_{2000}$ as a function of angular frequency at different temperatures in the range of -80 to $20{ }^{\circ} \mathrm{C}(\gamma=0.02 \%(-80$ to $\left.-40^{\circ} \mathrm{C}\right), 0.1 \%\left(-30^{\circ} \mathrm{C}\right), 1 \%\left(-20\right.$ to $\left.-10^{\circ} \mathrm{C}\right)$, and $10 \%\left(0\right.$ to $\left.\left.20^{\circ} \mathrm{C}\right)\right)$. The power laws $\omega^{2}$ and $\omega^{1}$ are indicative of terminal flow (black lines). 


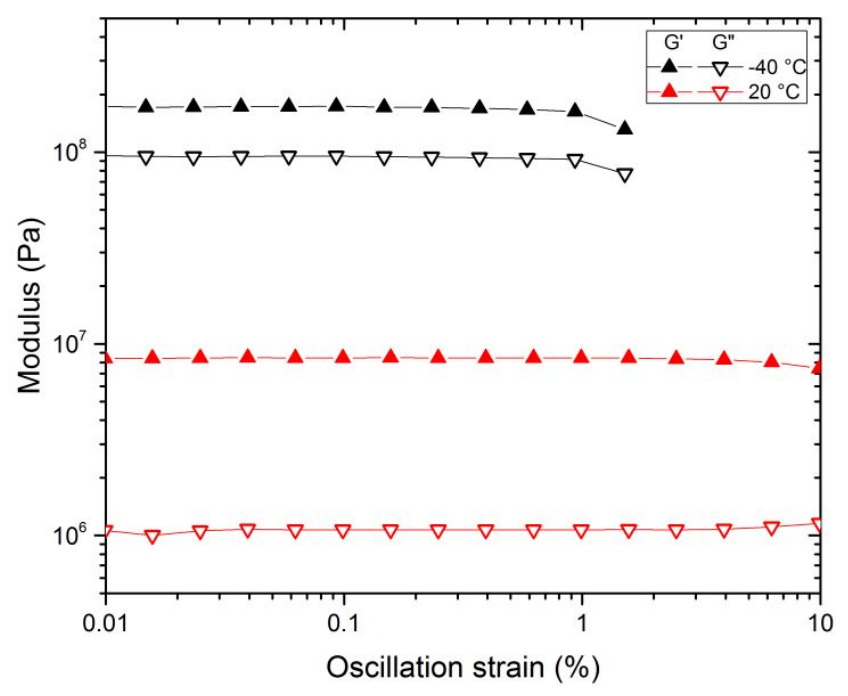

Figure S23. Plots of the modulus against the oscillation strain as determined by strain sweep experiments with poly(ethylene-co-butylene) macromonomer end-functionalized with Mebip-ligands $\left(\mathbf{B K}_{\mathbf{3 0 0 0}}\right)$ at a fixed angular frequency of $10 \%$. The measurements were employed to find the limit of the linear response behavior.
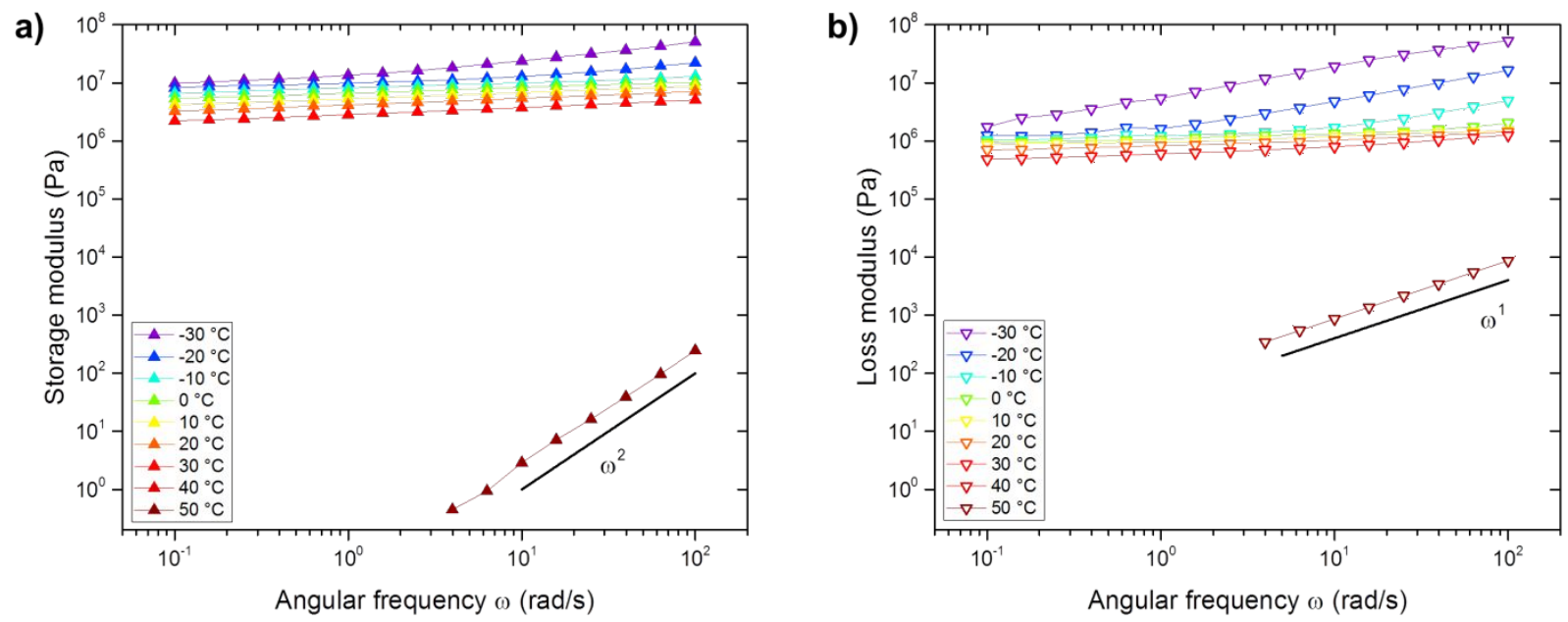

Figure S24. Linear shear rheology of the poly(ethylene-co-butylene) macromonomer end-functionalized with Mebip-ligands $\left(\mathbf{B K B}_{\mathbf{3 0 0 0}}\right)$. a) Storage and b) loss modulus as a function of angular frequency at different temperatures in the range of -30 to $50^{\circ} \mathrm{C}(\gamma=0.1 \%)$. At $50{ }^{\circ} \mathrm{C}$, the storage and loss moduli scale according to the power laws $\omega^{2}$ and $\omega^{1}$ respectively (black lines), indicative for terminal flow. 


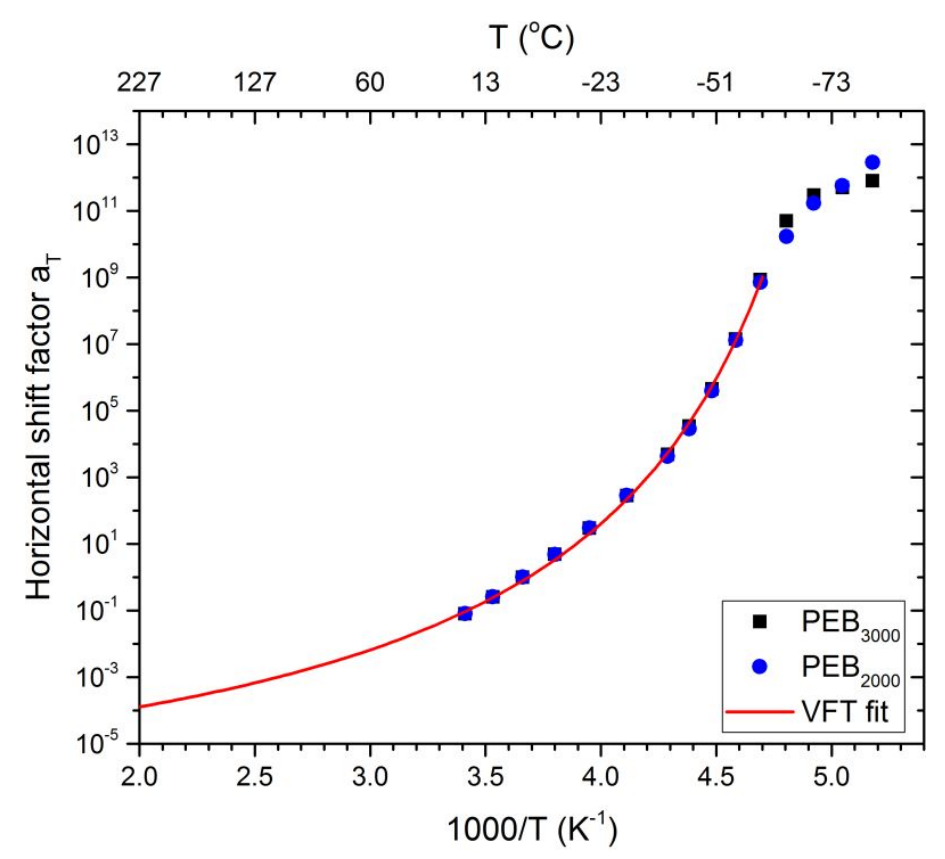

Figure S25. Horizontal shift factors $a_{\mathrm{T}}$ versus the inverse temperature of poly(ethylene-co-butylene) PEB ${ }_{3000}$ (black squares) and $\mathrm{PEB}_{2000}$ (blue circles), as well as the VFT-fit at temperatures above the $T_{\mathrm{g}}$ with an extrapolation towards higher temperatures (red line).

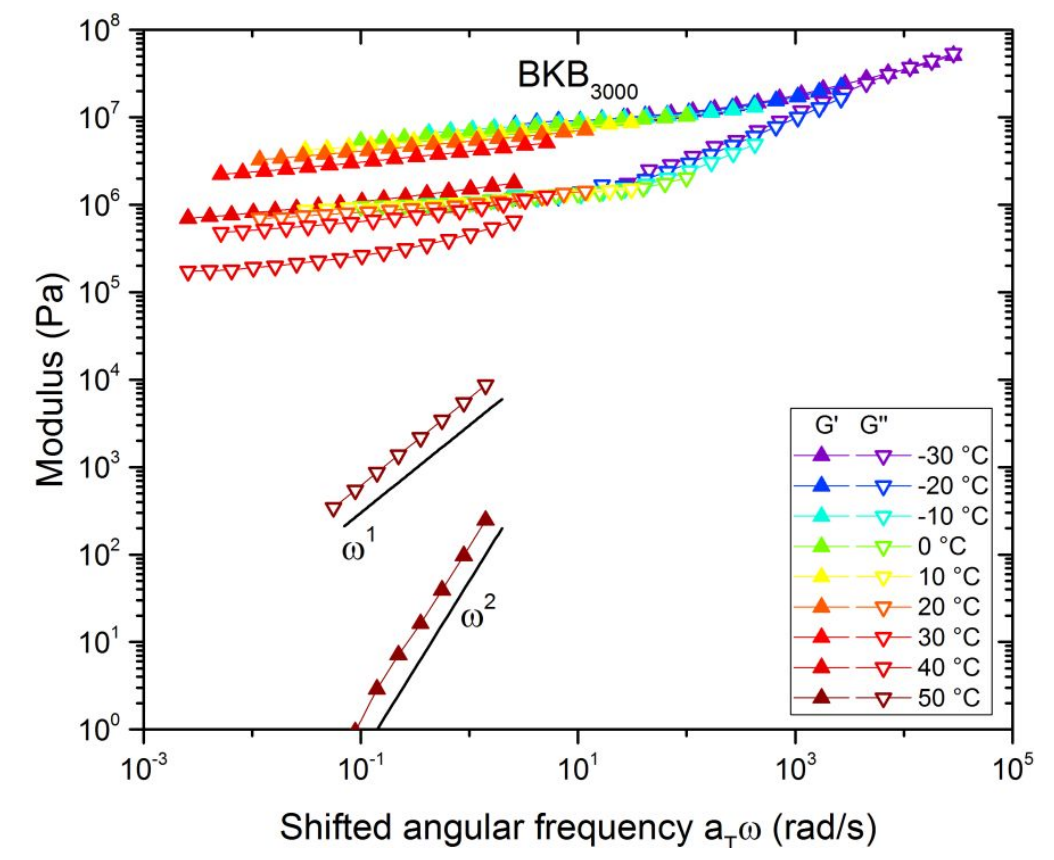

Figure S26. Linear shear rheology of the poly(ethylene-co-butylene) macromonomer $\mathbf{B K B}_{3000}$ end-functionalized with Mebip-ligands. Master curve of $\mathbf{B K B}_{3000}$ at $0{ }^{\circ} \mathrm{C}$ using extrapolated $\mathrm{PEB}_{3000}$ shift factors as shown in Figure S25. 

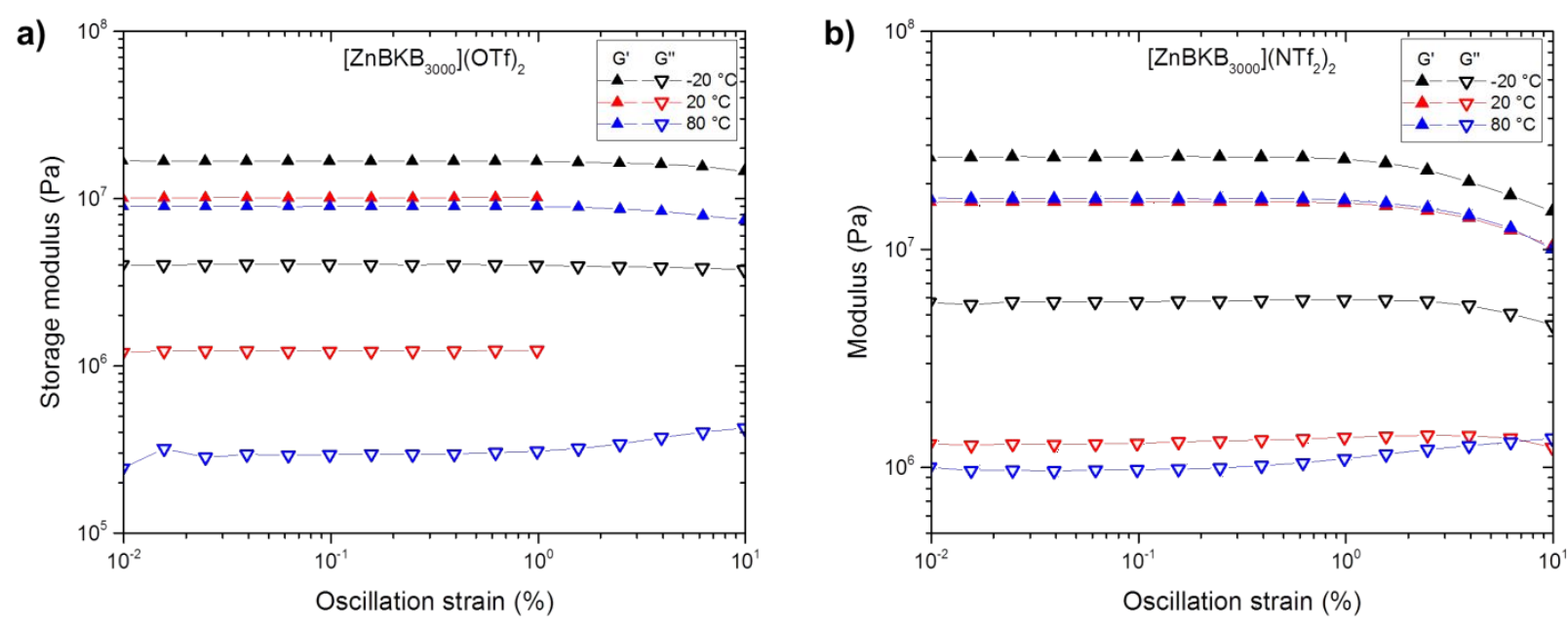

Figure S27. Plots of the modulus against the oscillation strain as determined by strain sweep experiments with the MSPs (a) $\left[\mathrm{ZnBKB}_{3000}\right](\mathrm{OTf})_{2}$ and (b) $\left[\mathrm{ZnBKB}_{3000}\right]\left(\mathrm{NTf}_{2}\right)_{2}$ at a fixed angular frequency of $10 \%$. The measurements were employed to determine the limit of the linear response behavior.

a)

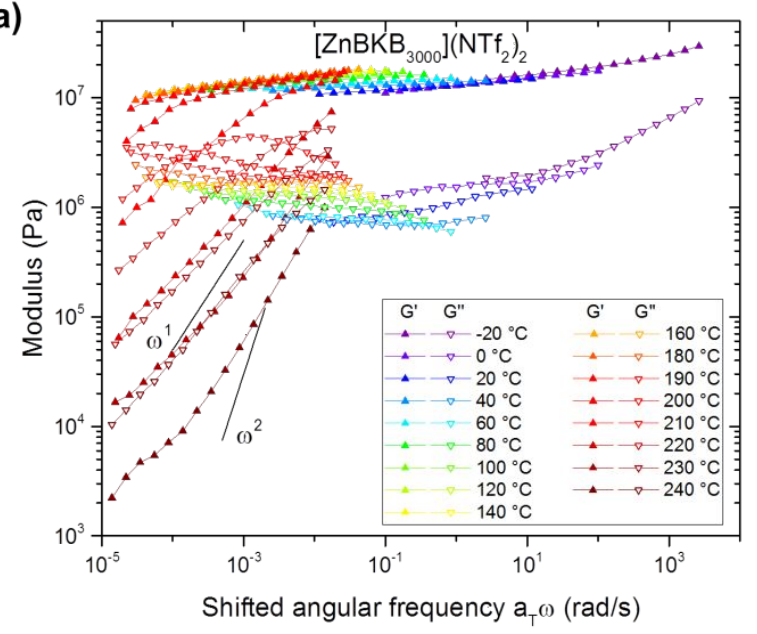

b)

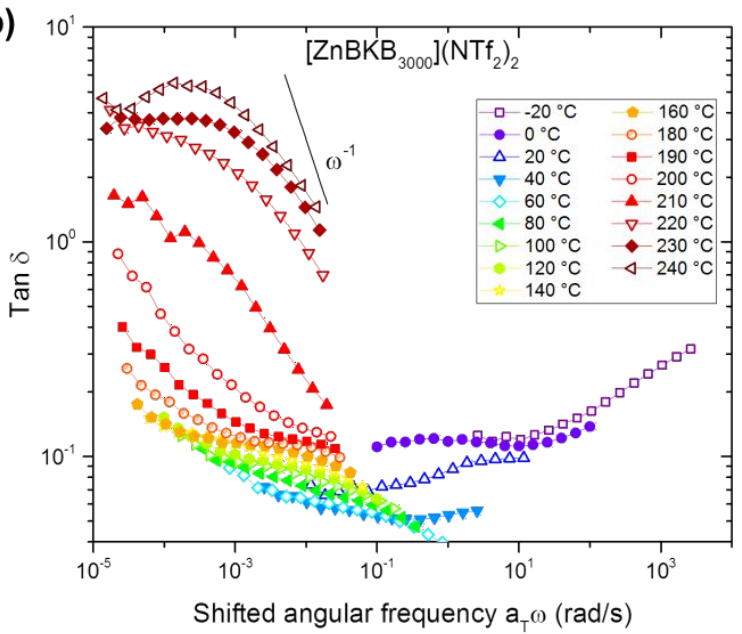

Figure S28. Linear shear rheology of $\left[\mathrm{ZnBKB}_{3000}\right]\left(\mathrm{NTf}_{2}\right)_{2}\left(\gamma=0.1 \%\left(-20\right.\right.$ to $\left.210^{\circ} \mathrm{C}\right), 1.0 \%\left(220\right.$ to $\left.\left.240{ }^{\circ} \mathrm{C}\right)\right)$. Master curves of (a) $G^{\prime}$ and $G^{\prime \prime}$, and (b) $\tan \delta$ at $T_{\text {ref }}=0^{\circ}{ }^{\circ} \mathrm{C}$ constructed using the extrapolated PEB $_{3000}$ shift factors as shown in Figure S25. The curves do not scale according to the power laws (black lines), indicating the absence of terminal flow in the material. 

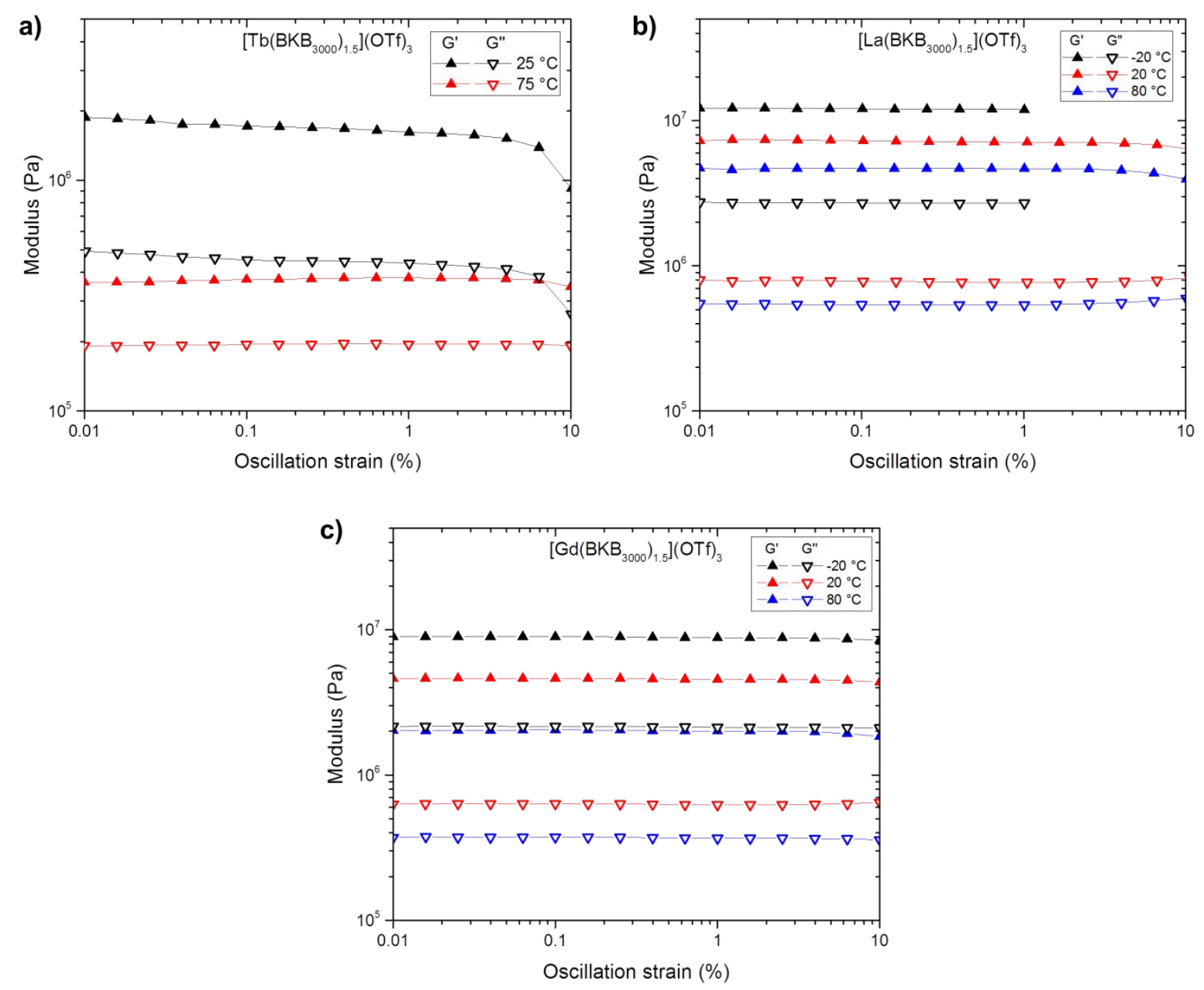

Figure 529. Strain sweep experiments at a fixed angular frequency of $10 \%$ to find the limit of the linear response behavior of (a) $\left.\left[\mathrm{Tb}\left(\mathrm{BKB}_{3000}\right)_{1.5}\right](\mathrm{OTf})_{3}, \mathrm{~b}\right)\left[\mathrm{La}\left(\mathrm{BKB}_{3000}\right)_{1.5}\right](\mathrm{OTf})_{3}$, and (c) $\left[\mathrm{Gd}\left(\mathrm{BKB}_{3000}\right)_{1.5}\right](\mathrm{OTf})_{3}$. 

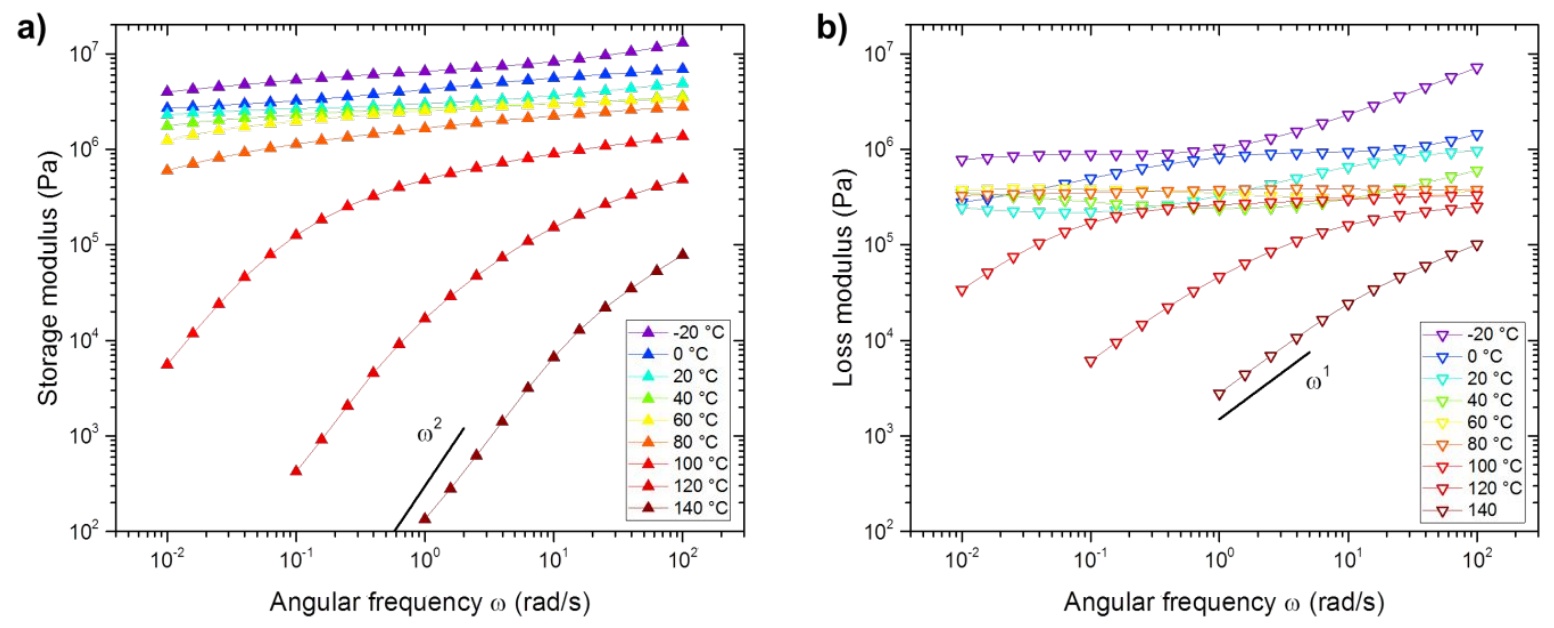

Figure S30. Linear shear rheology of the MSP $\left[\mathrm{Tb}\left(\mathrm{BKB}_{3000}\right)_{1.5}\right](\mathrm{OTf})_{3}$. a) Storage and (b) loss moduli as a function of angular frequency at different temperatures in the range of -20 to $140^{\circ} \mathrm{C}\left(\gamma=1.0 \%, \omega=0.01-100 \mathrm{rad} \mathrm{s}^{-1}\right)$.
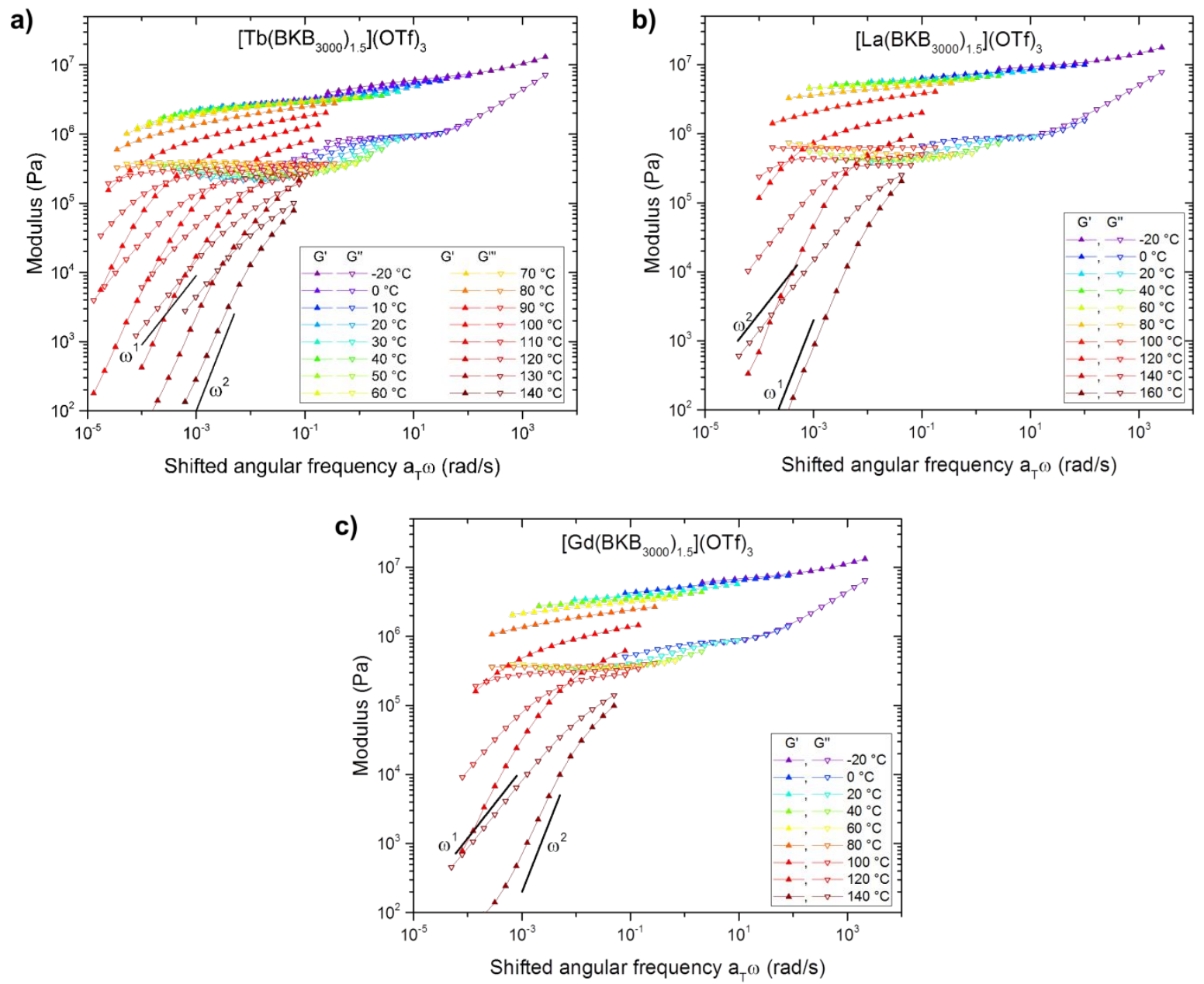

Figure S31. Linear shear rheology of the lanthanoid-based MSPs. Master curves of (a) $\left[\mathrm{Tb}\left(\mathrm{BKB}_{3000}\right)_{1.5}\right](\mathrm{OTf})_{3}$ $(\gamma=1.0 \%), b)\left[\operatorname{La}\left(\mathbf{B K B}_{3000}\right)_{1.5}\right](\mathrm{OTf})_{3}(\gamma=0.5 \%)$, and $(c)\left[\mathrm{Gd}\left(\mathrm{BKB}_{3000}\right)_{1.5}\right](\mathrm{OTf})_{3}(\gamma=0.5 \%)$ at $0{ }^{\circ} \mathrm{C}$ constructed using the $\mathrm{PEB}_{3000}$ shift factors as shown in Figure S25. The power laws $\omega^{1}$ and $\omega^{2}$ are plotted as well (black lines) to determine the region of terminal flow in the materials. 

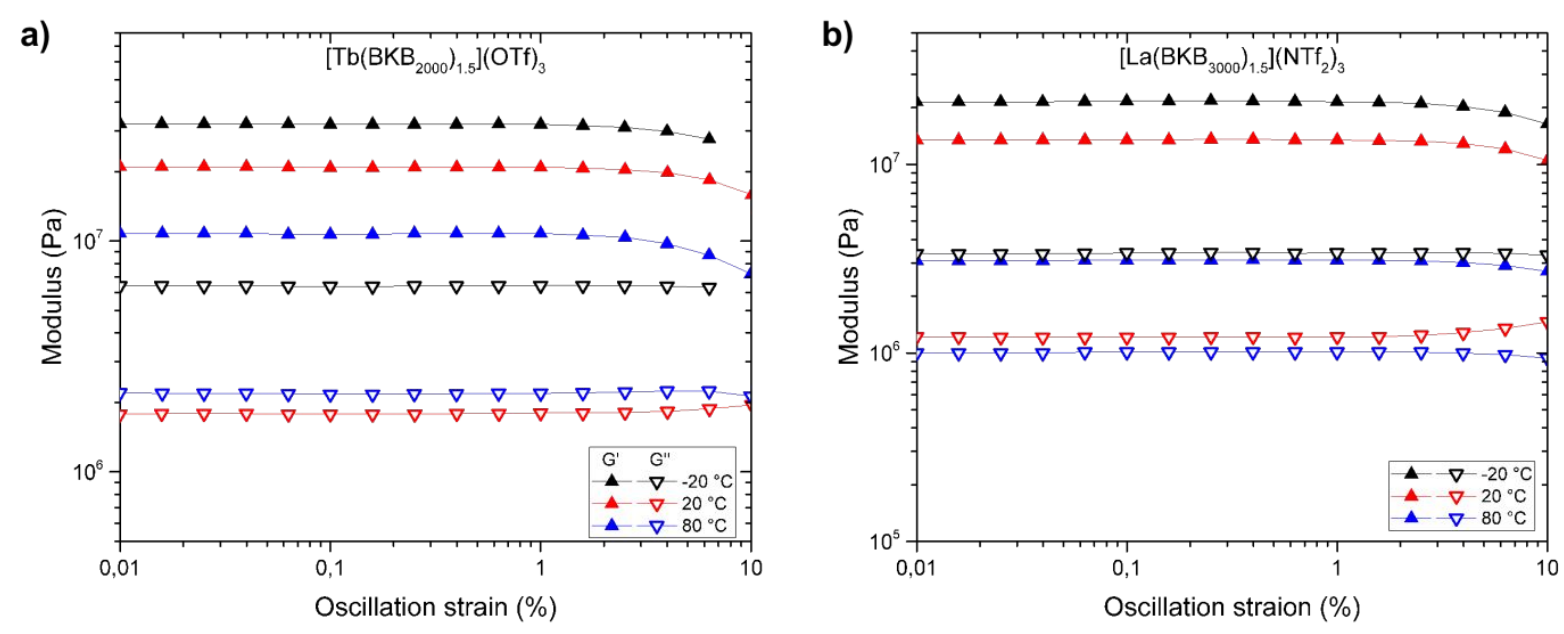

Figure S32. Plots of the modulus against the oscillation strain as determined by strain sweep experiments with the MSPs (a) $\left[\mathrm{Tb}\left(\mathrm{BKB}_{2000}\right)_{1.5}\right](\mathrm{OTf})_{3}$ and (b) $\left[\mathrm{La}\left(\mathrm{BKB}_{3000}\right)_{1.5}\right]\left(\mathrm{NTf}_{2}\right)_{3}$ at a fixed angular frequency of $10 \%$. The measurements were employed to determine the limit of the linear response behavior.
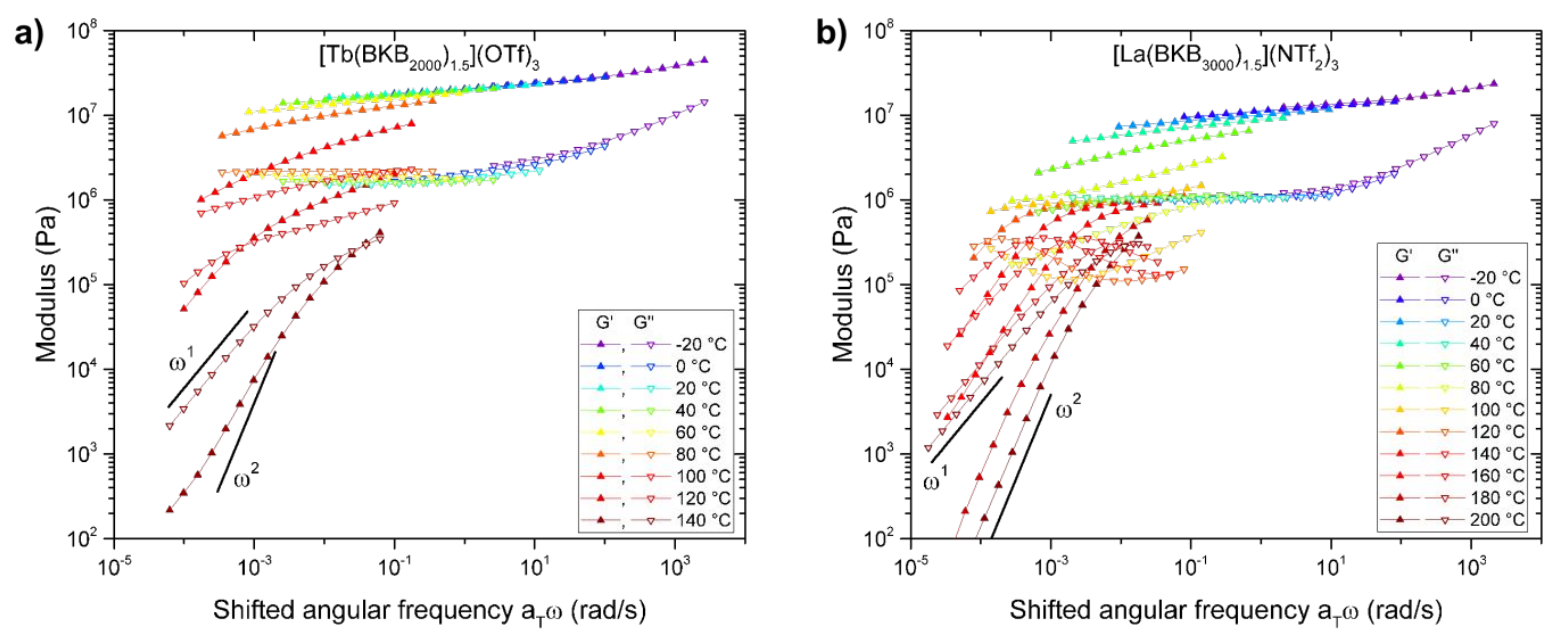

Figure S33. Linear shear rheology of the lanthanoid-based MSPs. Master curves of (a) $\left[\mathrm{Tb}\left(\mathbf{B K B}_{2000}\right)_{1.5}\right](\mathrm{OTf})_{3}$ and (b) $\left[\mathrm{La}\left(\mathrm{BKB}_{3000}\right)_{1.5}\right]\left(\mathrm{NTf}_{2}\right)_{3}$ at $0{ }^{\circ} \mathrm{C}$ constructed using the $\mathrm{PEB}_{3000}$ shift factors as shown in Figure $\mathrm{S} 25$. The power laws $\omega^{1}$ and $\omega^{2}$ are plotted as well (black lines) to determine the region of terminal flow in the materials. 
a)

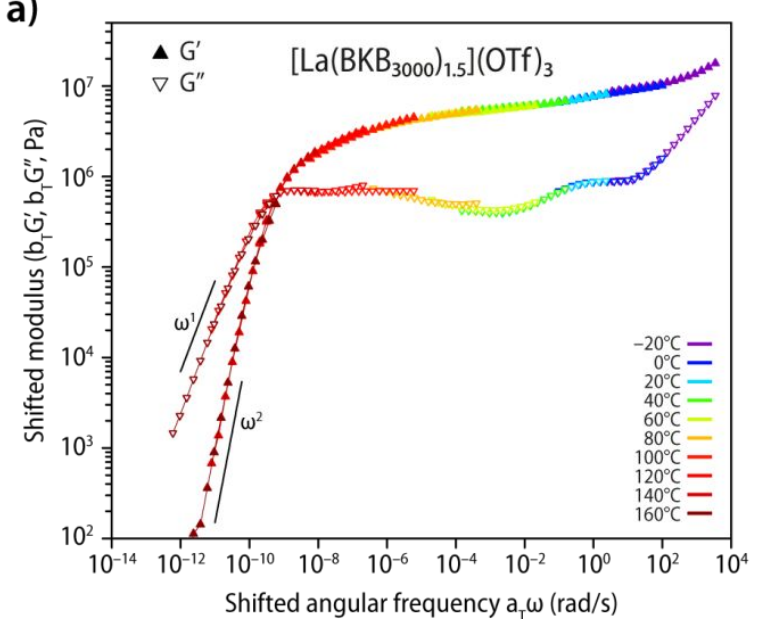

c)

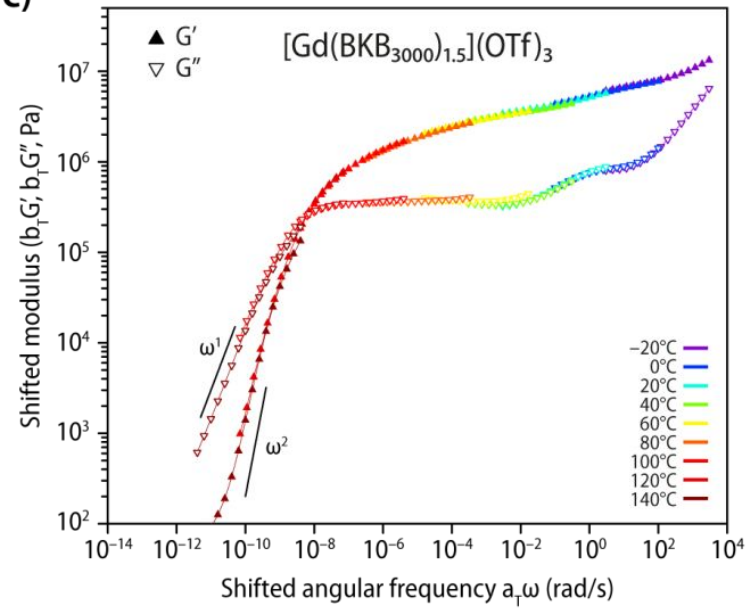

b)

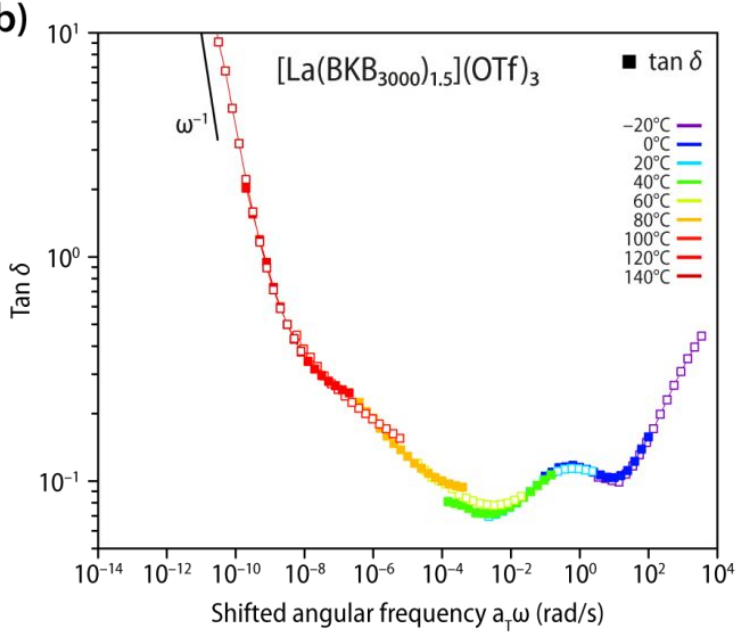

d)

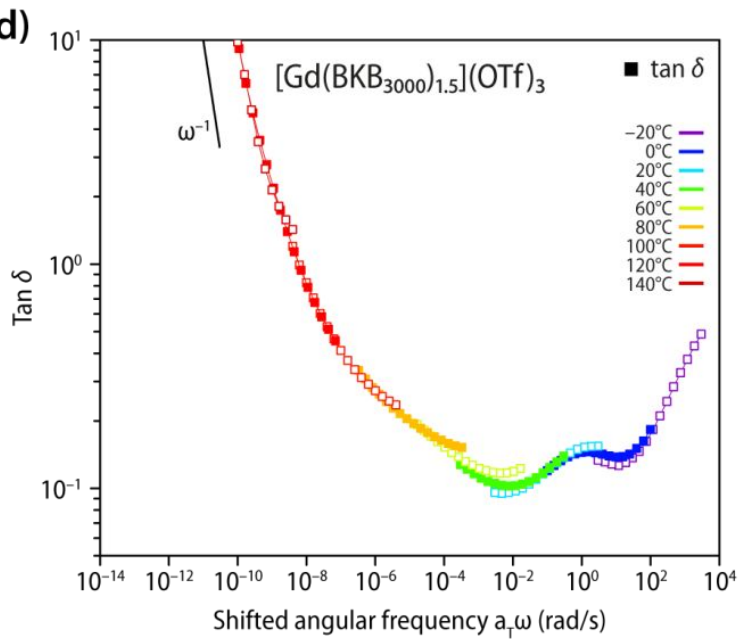

Figure S34. Linear shear rheology of lanthanoid-based MSPs. a-b) Master curves of [La(BKB $\left.\left.\mathbf{B}_{3000}\right)_{1.5}\right](\mathrm{OTf})_{3}$ for (a) $G^{\prime}$ and $G^{\prime \prime}$, and (b) $\tan \delta$ at $T_{\text {ref }}=0^{\circ} \mathrm{C}$ constructed using manual horizontal and vertical shift factors $a_{\mathrm{T}}$ and $b_{\mathrm{T}}$, respectively. c-d) Master curves of $\left.\mathrm{Gd}\left(\mathrm{BKB}_{3000}\right)_{1.5}\right](\mathrm{OTf})_{3}$ for (c) $G^{\prime}$ and $G^{\prime \prime}$, and (d) $\tan \delta$ at $T_{\text {ref }}=0^{\circ} \mathrm{C}$ constructed using manual horizontal and vertical shift factors $a_{\mathrm{T}}$ and $b_{\mathrm{T}}$, respectively. The power laws $\omega^{1}$ and $\omega^{2}$ are plotted as well (black lines) to determine the region of terminal flow in the materials. 

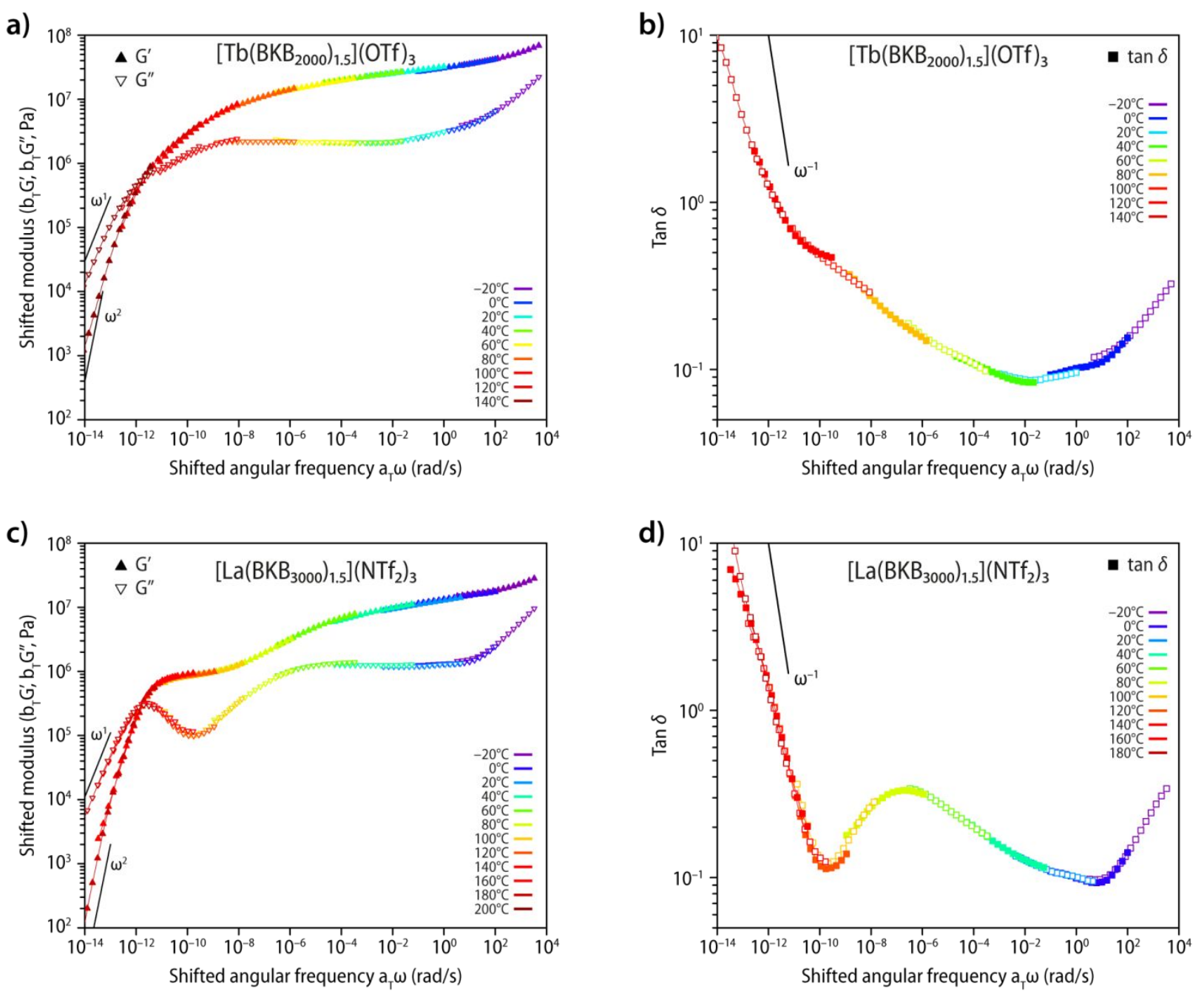

Figure S35. Linear shear rheology of lanthanoid-based MSPs. a-b) Master curves of $\left[\mathrm{Tb}\left(\mathbf{B K B}_{\mathbf{2 0 0 0}}\right)_{1.5}\right](\mathrm{OTf})_{3}$ for (a) $G^{\prime}$ and $G^{\prime \prime}$, and (b) $\tan \delta$ at $T_{\text {ref }}=0^{\circ} \mathrm{C}$ constructed using manual horizontal and vertical shift factors $a_{\mathrm{T}}$ and $b_{\mathrm{T}}$, respectively. c-d) Master curves of $\left[\mathrm{La}\left(\mathrm{BKB}_{3000}\right)_{1.5}\right]\left(\mathrm{NTf}_{2}\right)_{3}$ for (c) $G^{\prime}$ and $G^{\prime \prime}$, and (d) tan $\delta$ at $T_{\text {ref }}=0{ }^{\circ} \mathrm{C}$ constructed using manual horizontal and vertical shift factors $a_{\mathrm{T}}$ and $b_{\mathrm{T}}$, respectively. The power laws $\omega^{1}$ and $\omega^{2}$ are plotted as well (black lines) to determine the region of terminal flow in the materials. 


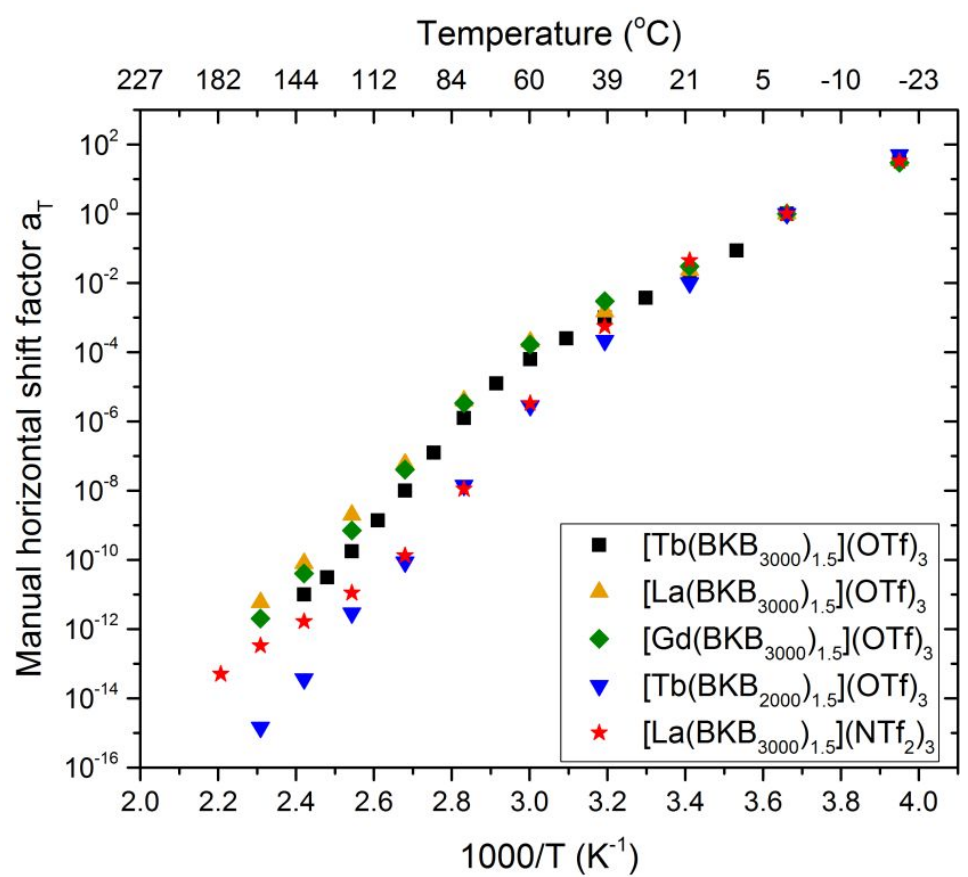

Figure S36. Plot of the manual horizontal shift factors $\left(a_{T}\right)$ as a function of inverse temperature of the MSPs $\left[\mathrm{Tb}\left(\mathbf{B K B}_{\mathbf{3 0 0 0}}\right)_{1.5}\right](\mathrm{OTf})_{3}$ (black squares), $\left[\mathrm{La}\left(\mathbf{B K B}_{3000}\right)_{1.5}\right](\mathrm{OTf})_{3}$ (orange triangles), $\left[\mathrm{Gd}\left(\mathrm{BKB}_{3000}\right)_{1.5}\right](\mathrm{OTf})_{3}$ (green diamonds), $\left[\mathrm{Tb}\left(\mathbf{B K B}_{2000}\right)_{1.5}\right](\mathrm{OTf})_{3}$ (blue triangles), and [ $\left.\mathbf{L a}\left(\mathbf{B K B}_{3000}\right)_{1.5}\right]\left(\mathrm{NTf}_{2}\right)_{3}$ (red stars). 


\section{Supplementary Tables S1-S2}

Table S1. Overview of the thermal transitions as observed by differential scanning calorimetry (DSC) for selected metal-ligand complexes with the model compound bis(1'-methylbenzimidazoyl)-4-(2-ethylhexyloxy)pyridine (MC).

\begin{tabular}{|c|c|c|c|}
\hline Compound & $T_{\mathrm{g}}\left({ }^{\circ} \mathrm{C}\right)$ & $T_{\mathrm{m}}\left({ }^{\circ} \mathrm{C}\right)$ & $T_{\mathrm{c}}\left({ }^{\circ} \mathrm{C}\right)$ \\
\hline MC & & $152-156$ & 109 \\
\hline$\left[\mathrm{Zn}(\mathrm{MC})_{2}\right](\mathrm{OTf})_{2}$ & & 314 & 141 \\
\hline$\left[\mathrm{Zn}(\mathrm{MC})_{2}\right]\left(\mathrm{NTf}_{2}\right)_{2}$ & & 241 & 126 \\
\hline$\left[\mathrm{Tb}(\mathrm{MC})_{3}\right](\mathrm{OTf})_{3}$ & 137 & 171 & \\
\hline$\left[\mathrm{La}(\mathbf{M C})_{3}\right]\left(\mathrm{NTf}_{2}\right)_{3}$ & 100 & 150 & \\
\hline
\end{tabular}

All values as observed in the first heating and cooling cycle $\left(10^{\circ} \mathrm{C} \mathrm{min}^{-1}\right)$.

Table S2. Volume of the hard- and soft phase of the different MSPs as estimated via computational analysis. The values were used to calculate the volume fraction of the hard- and soft phase.

\begin{tabular}{lc}
\hline Hard phase & Volume $\left(\AA^{3}\right)$ \\
\hline \hline$\left[\mathrm{M}^{2+}\right.$ Mebip $\left._{2}\right](\mathrm{OTf})_{2}$ & 887 \\
{$\left[\mathrm{M}^{2+} \mathrm{Mebip}_{2}\right]\left(\mathrm{NTf}_{2}\right)_{2}$} & 1027 \\
{$\left[\mathrm{M}^{2+} \mathrm{Mebip}_{2}\right]\left(\mathrm{ClO}_{4}\right)_{2}$} & 827 \\
\hline$\left[\mathrm{M}^{3+} \mathrm{Mebip}_{3}\right](\mathrm{OTf})_{3}$ & 1313 \\
{$\left[\mathrm{M}^{3+} \mathrm{Mebip}_{3}\right]\left(\mathrm{NTf}_{2}\right)_{3}$} & 1527 \\
{$\left[\mathrm{M}^{3+} \mathrm{Mebip}_{3}\right]\left(\mathrm{ClO}_{4}\right)_{3}$} & 1223 \\
\hline Soft Phase & Volume $\left(\AA^{3}\right)$ \\
\hline \hline PEB & \\
\hline PEB & 4016 \\
\hline
\end{tabular}




\section{Experimental Details}

\subsection{Instrumentation}

NMR Spectroscopy. NMR spectra were recorded at $297.2 \mathrm{~K}$ on a Bruker Avance DPX 400 spectrometer at frequencies of $400.19 \mathrm{MHz}$ for ${ }^{1} \mathrm{H}$ nuclei and $100.63 \mathrm{MHz}$ for ${ }^{13} \mathrm{C}$ nuclei. Spectra were calibrated to the residual solvent peak of $\mathrm{CDCl}_{3}$ (7.26 ppm ${ }^{1} \mathrm{H}$ NMR; $77.16 \mathrm{ppm}{ }^{13} \mathrm{C} \mathrm{NMR}$ ) or DMSO- $\mathrm{d}_{6}$ (2.50 ppm ${ }^{1} \mathrm{H}$ NMR; $39.52 \mathrm{ppm}{ }^{13} \mathrm{C}$ NMR). Data were treated with MestReNova (12.0.2) software suite and all chemical shifts $\delta$ are reported in parts per million (ppm) relative to tetramethyl silane.

Size exclusion chromatography (SEC). SEC analysis was conducted on an Agilent 1200 series HPLC system equipped with two Agilent PLgel mixed-D columns (ID $=7.5 \mathrm{~mm}, \mathrm{~L}=300 \mathrm{~mm}$, particle size $=5 \mu \mathrm{m}$ ) and an Agilent PLgel mixed guard column (particle size $=5 \mu \mathrm{m}$ ) at $30{ }^{\circ} \mathrm{C}$ and a flow rate of $1.0 \mathrm{~mL} \mathrm{~min}{ }^{-1}$ using THF as the eluent. Signals were acquired by an Optilab REX interferometric refractometer and data was processed using the Astra software (Wyatt Technology Corp.).

UV-vis spectroscopy. Solution phase UV-vis spectra were recorded on a Shimadzu UV-2401 PC spectrophotometer using quartz cuvettes of $1 \mathrm{~cm}$ path length.

Thermogravimetric analysis (TGA). Measurements were conducted in air using a Mettler-Toledo STAR thermogravimetric analyzer in the range of $25^{\circ} \mathrm{C}$ to $500{ }^{\circ} \mathrm{C}$ with a heating rate of $10^{\circ} \mathrm{C} \mathrm{min}^{-1}$.

Differential scanning calorimetry (DSC). Measurements were performed under $\mathrm{N}_{2}$ using a Mettler-Toledo STAR system operating at a heating/cooling rate of $10{ }^{\circ} \mathrm{C} \mathrm{min}^{-1}$ in the range of -80 to $200{ }^{\circ} \mathrm{C}$, unless indicated otherwise. The midpoint of the step change in the heat capacity is reported as the glass transition temperature $\left(T_{g}\right)$, the melting temperature $\left(T_{m}\right)$ is reported based on the minimum of the major endothermic melting peak, and the crystallization temperature $\left(T_{c}\right)$ is reported based on the maximum of the major exothermic crystallization peak.

Dynamic mechanical analyses (DMA). Measurements were conducted under $\mathrm{N}_{2}$ on a TA Instruments DMA Q800 with a heating rate of $3^{\circ} \mathrm{C} \mathrm{min}^{-1}$, a frequency of $1 \mathrm{~Hz}$, and an amplitude of $15 \mu \mathrm{m}$ in the range of -80 to $300{ }^{\circ} \mathrm{C}$, using tensile clamps and rectangular shaped samples. The reported mechanical data are averages of 3 independent experiments, and all errors are standard deviations.

Small-angle and wide-angle X-ray scattering (SAXS/WAXS). Experiments were performed on a Rigaku NanoMax camera (Rigaku Innovative Technologies, Auburn Hills, MI). The camera was equipped with a Cu target sealed tube source (MicroMax 003 microfocus, Rigaku), and the scattering data were recorded by a Pilatus100 K detector (Dectris). The sample-to-detector distance was calibrated using silver behenate. For the temperature-dependent experiments a Linkam HFS-X350-GI heating stage module with a T95 
controller was used. Stainless-steel discs with a thickness of $2 \mathrm{~mm}$ and a central cylindrical hole (diameter of $2 \mathrm{~mm}$ ) were employed as samples holders that were mounted onto the heating stage. Samples were cut into pieces of appropriate size and pressed into the sample holder. The heating and cooling rate for temperature-dependent scattering experiments was $10^{\circ} \mathrm{C} \mathrm{min}^{-1}$, and samples were equilibrated at each temperature for $10 \mathrm{~min}$ before data collection was started. Raw data were processed according to standard procedures. Scattering spectra are presented as a function of the momentum transfer $q=4 \pi \lambda^{-1}$ $\sin (\theta / 2)$, where $\theta$ is the scattering angle and $\lambda=0.1524 \mathrm{~nm}$ is the photon wavelength.

Scanning transmission electron microscopy (STEM). Imaging was performed on a Thermo Scientific Titan Themis 60-300 transmission electron microscope operated at $200 \mathrm{kV}$ in scanning mode and using highangle annular dark-field conditions (STEM-HAADF). The microscope is equipped with a high brightness XFEG gun and silicon drift Super-X EDX detectors and Velox acquisition software. Samples for STEM analysis were prepared by cryo-ultramicrotomy. Sample were ultramicrotomed at $-50^{\circ} \mathrm{C}$ with a Leica EM FC7 machine (equipped with a Diatome cryo-knife $35^{\circ}$ angle) at a cutting speed of $0.2 \mathrm{~mm} \mathrm{~s}$. Ultramicrotomed slices were deposited on an ultra-thin carbon support grid.

Optical microscopy (OM) and polarized optical microscopy (POM). Images were acquired with an Olympus BX51 microscope equipped with a DP72 digital camera and polarized optical microscopy images were collected with crossed polarizers. For temperature-dependent experiments, a Linkam LTS 350 heating stage module was used. Heating and cooling rates of $10^{\circ} \mathrm{C} \mathrm{min}^{-1}$ were used in the temperature regime where thermal transitions were observed in the DSC traces, and heating and cooling rates of $30^{\circ} \mathrm{C}$ $\mathrm{min}^{-1}$ were used outside these regimes.

Rheological measurements. The measurements were performed using either an Anton Paar MCR 501 rheometer connected to a Peltier controlled measurement system in parallel plate geometry and a dry $\mathrm{N}_{2}$ stream for temperature control, or an ARES-G2 from TA-Instruments with a constant $\mathrm{N}_{2}$ gas stream for temperature control. A geometry with parallel plates with a diameter of $25 \mathrm{~mm}$ or $8 \mathrm{~mm}$ was used. All lanthanide films were equilibrated in the melt at $160^{\circ} \mathrm{C}\left(\left[\mathrm{Tb}\left(\mathrm{BKB}_{3000}\right)_{1.5}\right](\mathrm{OTf})_{3},\left[\mathrm{~Tb}\left(\mathrm{BKB}_{2000}\right)_{1.5}\right](\mathrm{OTf})_{3}\right.$, $\left[\mathrm{La}\left(\mathrm{BKB}_{3000}\right)_{1.5}\right](\mathrm{OTf})_{3}$, and $\left.\left[\mathrm{Gd}\left(\mathrm{BKB}_{3000}\right)_{1.5}\right](\mathrm{OTf})_{3}\right)$ or $180{ }^{\circ} \mathrm{C}\left(\left[\mathrm{La}\left(\mathrm{BKB}_{3000}\right)_{1.5}\right]\left(\mathrm{NTf}_{2}\right)_{3}\right)$ before the start of the measurement to remove any thermal history from the compression-molding step. For MSP films containing $\mathrm{Zn}^{2+}$ ions, samples were employed as obtained by compression-molding as an annealing in the melt was not possible because the melting temperature is around the onset of thermal degradation in the materials. The sample thickness was between 0.4 and $1.0 \mathrm{~mm}$. The limit for linear response behavior was checked first. Afterwards, rheological measurements in the linear range were done in a wide range of temperatures and angular frequencies. Measurements at each temperature were performed after an equilibration time of at least $5 \mathrm{~min}$ at an angular frequency range of 100-0.1 rad s${ }^{-1}$ unless otherwise indicated. Time-temperature superposition was performed by shifting the frequency-dependent 
oscillatory $G^{\prime}$ or $G^{\prime \prime}$ shear moduli or the loss tangent $(\tan \delta)$ horizontally along the frequency axis to a reference temperature $\left(T_{\text {ref }}\right)$ of $0{ }^{\circ} \mathrm{C}$ until good overlap was achieved. If necessary, for a good overlap of $G^{\prime}$ and $G^{\prime \prime}$, the data was subsequently shifted along the horizontal axis by a vertical shift factor $b_{\mathrm{T}}$.

The scaling of the horizontal shift factors $a_{\mathrm{T}}$ was fitted to the VFT-model: ${ }^{5}$

$$
a_{T}=a_{T, 0} 10^{\left(A+\frac{B}{T-T_{0}}\right)} \quad \text { Equation S1 }
$$

where $A$ and $B$ represent fitting constants, $a_{\mathrm{T}, 0}$ represents the horizontal shift factor at the reference temperature, $T$ is the temperature in $\mathrm{K}$, and $T_{0}$ is the Vogel temperature that is typically $50^{\circ} \mathrm{C}$ below $T_{\mathrm{g}}$.

The apparent activation energies were fitted according to the following equations: ${ }^{6}$

$$
\begin{array}{ll}
\operatorname{Ln}\left(a_{T}\right)=B_{0}+\frac{E_{a}}{R T} & \text { Equation S2 } \\
\operatorname{Ln}\left(\tau_{b}\right)=A_{0}+\frac{E_{a}}{R T} & \text { Equation S3 }
\end{array}
$$

where $\tau_{\mathrm{b}}$ represents the supramolecular bond lifetime determined as the crossover point of $\mathrm{G}^{\prime}$ and $\mathrm{G}^{\prime \prime}$, $a_{\mathrm{T}}$ are the manual horizontal shift factors, $E_{\mathrm{a}}$ is the apparent activation energy in $\mathrm{J} \mathrm{mol}^{-1}, R$ is the gas constant $8.3145 \mathrm{~J} \mathrm{~K}^{-1} \mathrm{~mol}^{-1}$, and $T$ is the temperature in $\mathrm{K}$.

\subsection{Materials and Methods}

UV-vis spectrophotometric titrations. To demonstrate that the prepared macromonomer forms complexes in the same manner as previously reported UV-vis absorption spectra were acquired upon titration of solutions of the macromonomers $\mathbf{B K B}_{3000}$ and $\mathbf{B K B}_{2000}\left(c=10 \mu \mathrm{mol} \mathrm{L}{ }^{-1}\right.$ in $\mathrm{CHCl}_{3} / \mathrm{MeCN}$ 9:1) with aliquots of $\mathrm{Zn}\left(\mathrm{NTf}_{2}\right)_{2}\left(c=230 \mu \mathrm{mol} \mathrm{L} \mathrm{L}^{-1}\right.$ in $\mathrm{CHCl}_{3} / \mathrm{MeCN}$ 9:1, containing also the respective BKB $(c=10 \mu \mathrm{mol}$ $\mathrm{L}^{-1}$ to avoid dilution). Moreover, UV-vis absorption spectra were acquired upon titrations of the low molecular weight model compound $\mathrm{MC}\left(c=25 \mu \mathrm{mol} \mathrm{L}^{-1}\right)$ in anhydrous $\mathrm{MeCN}$ with $\mathrm{Zn}(\mathrm{OTf})_{2}(c=$ $\left.303 \mu \mathrm{mol} \mathrm{L}^{-1}\right)$ or $\mathrm{Tb}(\mathrm{OTf})_{3}\left(c=208 \mu \mathrm{mol} \mathrm{L}^{-1}\right)$ in anhydrous MeCN, unless otherwise indicated. In this case, the solutions contained $\mathbf{M C}\left(c=25 \mu \mathrm{mol} \mathrm{L}^{-1}\right)$ to avoid dilution effects. The characteristic absorbance band for the ligand and the formed $\mathrm{Zn}^{2+}$ - or $\mathrm{Tb}^{3+}$-complexes were plotted as a function of the metal-to-ligand ratio.

Metallosupramolecular polymerization and film formation. To a stirred solution of the BKB macromonomer in $\mathrm{CHCl}_{3}$, the proper amount of a solution of the metal salt in anhydrous $\mathrm{MeCN}$ was added to ensure a stoichiometric metal-to-ligand ratio. The solution was stirred for 10 minutes, the solvent was removed in vacuo and the sample was redissolved in $\mathrm{CHCl}_{3}$. The solution was dried in vacuo, and placed in a vacuum oven overnight at $40{ }^{\circ} \mathrm{C}$. Subsequently, the samples of the metallosupramolecular polymers 
were compression molded at different temperatures with a pressure of 3 tons for $4 \mathrm{~min}$. The compressionmolding was carried out at $200{ }^{\circ} \mathrm{C}$ for $\left(\left[\mathrm{ZnBKB}_{3000}\right](\mathrm{OTf})_{2}\right.$, $\left[\mathrm{ZnBKB}_{2000}\right](\mathrm{OTf})_{2}$, $\left[\mathrm{FeBKB}{ }_{3000}\right](\mathrm{OTf})_{2}$, and $\left.\left[\mathrm{FeBKB}_{2000}\right](\mathrm{OTf})_{2}\right)$, at $140^{\circ} \mathrm{C}$ for samples of $\left(\left[\mathrm{ZnBKB}_{3000}\right]\left(\mathrm{ClO}_{4}\right)_{2}\right.$, $\left[\mathrm{FeBKB}{ }_{3000}\right]\left(\mathrm{ClO}_{4}\right)_{2}$, $\left[\mathrm{FeBKB}_{2000}\right]\left(\mathrm{ClO}_{4}\right)_{2}$, $\left[\mathrm{ZnBKB}_{3000}\right]\left(\mathrm{NTf}_{2}\right)_{2},\left[\mathrm{ZnBKB}_{2000}\right]\left(\mathrm{NTf}_{2}\right)_{2}$, and $\left[\mathrm{La}\left(\mathrm{BKB}_{3000}\right)_{1.5}\right]\left(\mathrm{NTf}_{2}\right)_{3}$, or at temperatures of $100{ }^{\circ} \mathrm{C}$ for samples of $\quad\left(\left[\mathrm{Tb}\left(\mathrm{BKB}_{3000}\right)_{1.5}\right](\mathrm{OTf})_{3}, \quad\left[\mathrm{~Tb}\left(\mathrm{BKB}_{2000}\right)_{1.5}\right](\mathrm{OTf})_{3}, \quad\left[\mathrm{La}\left(\mathrm{BKB}_{3000}\right)_{1.5}\right](\mathrm{OTf})_{3}, \quad\left[\mathrm{~Tb}\left(\mathrm{BKB}_{3000}\right)_{1.5}\right]\left(\mathrm{ClO}_{4}\right)_{3}\right.$, $\left[\mathrm{Tb}\left(\mathrm{BKB}_{2000}\right)_{1.5}\right]\left(\mathrm{ClO}_{4}\right)_{3},\left[\mathrm{Gd}\left(\mathrm{BKB}_{3000}\right)_{1.5}\right](\mathrm{OTf})_{3}$, as well as $\left[\mathrm{Gd}\left(\mathrm{BKB}_{3000}\right)_{1.5}\right]\left(\mathrm{ClO}_{4}\right)_{3}$.

Formation of metal-ligand complexes with model ligand. To a stirred solution of the ligand $\mathbf{M C}$ in $\mathrm{CHCl}_{3}$, a proper amount of a solution of the metal salt in anhydrous MeCN was added to ensure a stoichiometric metal-to-ligand ratio. The solution was stirred for $10 \mathrm{~min}$, and subsequently dried under an $\mathrm{N}_{2}$ stream and then in a vacuum oven overnight at $40^{\circ} \mathrm{C}$.

Computational analysis of the volume fraction. A geometry optimization was performed with the semiempirical PM3 model as implemented in the Spartan 16 software suite. All thresholds were left to the default settings and the provided values for the CPK volume of the different complexes are those obtained after structure optimization.

\subsection{Synthetic Procedures and Analytical Data}

General Procedures. Poly(ethylene-co-butylene) (Krasol ${ }^{\circledR}$ ) of a number-average molecular weight of 3100 and $2100 \mathrm{~g} \mathrm{~mol}^{-1}$ was obtained from Cray Valley HSC and dried in vacuo at $40{ }^{\circ} \mathrm{C}$ overnight prior to use. Spectroscopy grade chloroform (Acros organics) was passed over dry, activated (Brockman I) basic alumina prior to use to remove acidic impurities. Zinc(II) trifluoromethanesulfonate $\left(\mathrm{Zn}(\mathrm{OTf})_{2}\right)$ (Aldrich), Zinc(II) perchlorate hexahydrate $\left(\mathrm{Zn}\left(\mathrm{ClO}_{4}\right)_{2} \cdot 6 \mathrm{H}_{2} \mathrm{O}\right)$ (Strem), Zinc bis(trifluoromethylsulfonyl)imide $\left(\mathrm{Zn}\left(\mathrm{NTf}_{2}\right)_{2}\right)$ (Aldrich), Iron(II) trifluoromethanesulfonate (Fe(OTf $\left.)_{2}\right)$ (Aldrich), Iron(II) perchlorate hexahydrate $\left(\mathrm{Fe}\left(\mathrm{ClO}_{4}\right)_{2} \cdot 6 \mathrm{H}_{2} \mathrm{O}\right)$ (Aldrich), Terbium trifluoromethanesulfonate (Tb(OTf) $\left.)_{3}\right)$ (Aldrich), Terbium perchlorate $\left(\mathrm{Tb}\left(\mathrm{ClO}_{4}\right)_{3} ; 50 \%\right.$ aqueous solution) (Strem), Gadolinium trifluoromethanesulfonate $\left(\mathrm{Gd}(\mathrm{OTf})_{3}\right)$ (Aldrich), Gadolinium perchlorate $\left(\mathrm{Gd}\left(\mathrm{ClO}_{4}\right)_{3}\right)$ (50\% aqueous solution) (Strem), Lanthanum bis(trifluoromethanesulfonyl)imide $\left(\mathrm{La}\left(\mathrm{NTf}_{2}\right)_{3}\right)(\mathrm{TCl})$, anhydrous MeCN (Acros organics), and all other reagents were commercially obtained and used without further purification.

The 2,6-bis(1'-methylbenzimidazolyl)pyridine (Mebip) ligand, the bis(1'-methylbenzimidazoyl)-4-(2-ethylhexyloxy)pyridine MC as a low molecular weight model compound, and the bis(Mebip)-functionalized telechelic poly(ethylene-co-butylene) (BKB) macromonomers $\mathrm{BKB}_{3000}$ and $\mathrm{BKB}_{2000}$ were prepared following previously reported procedures. ${ }^{1,2,7}$ 


\subsection{NMR and MALDI spectra}

${ }^{1} \mathrm{H}$ and ${ }^{13} \mathrm{C}$ NMR Spectra $\left(\mathrm{CDCl}_{3}, 400 \mathrm{MHz}\right)$ of $\mathrm{BKB}_{3000}$.
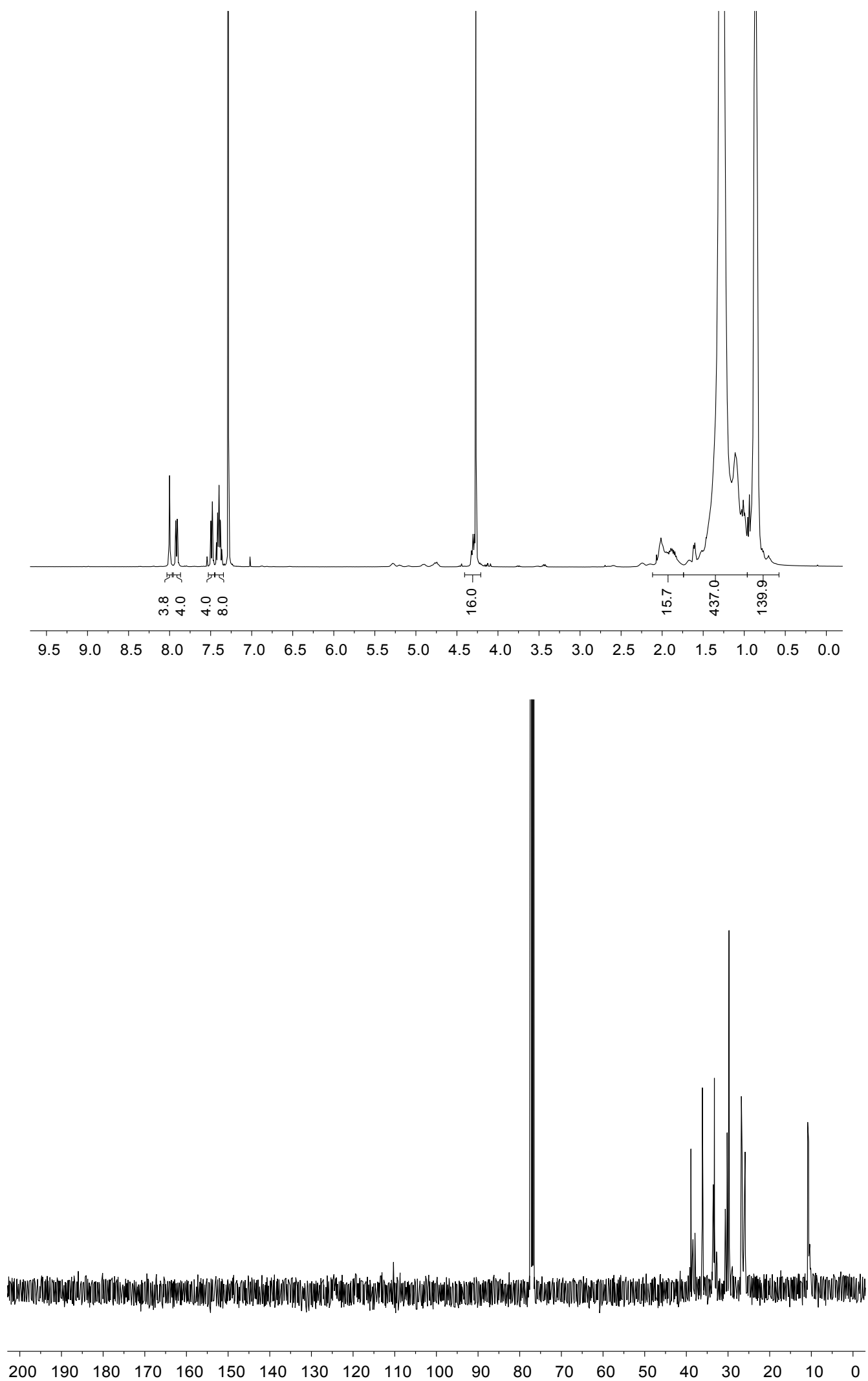
MALDI of $B B_{3000}$.

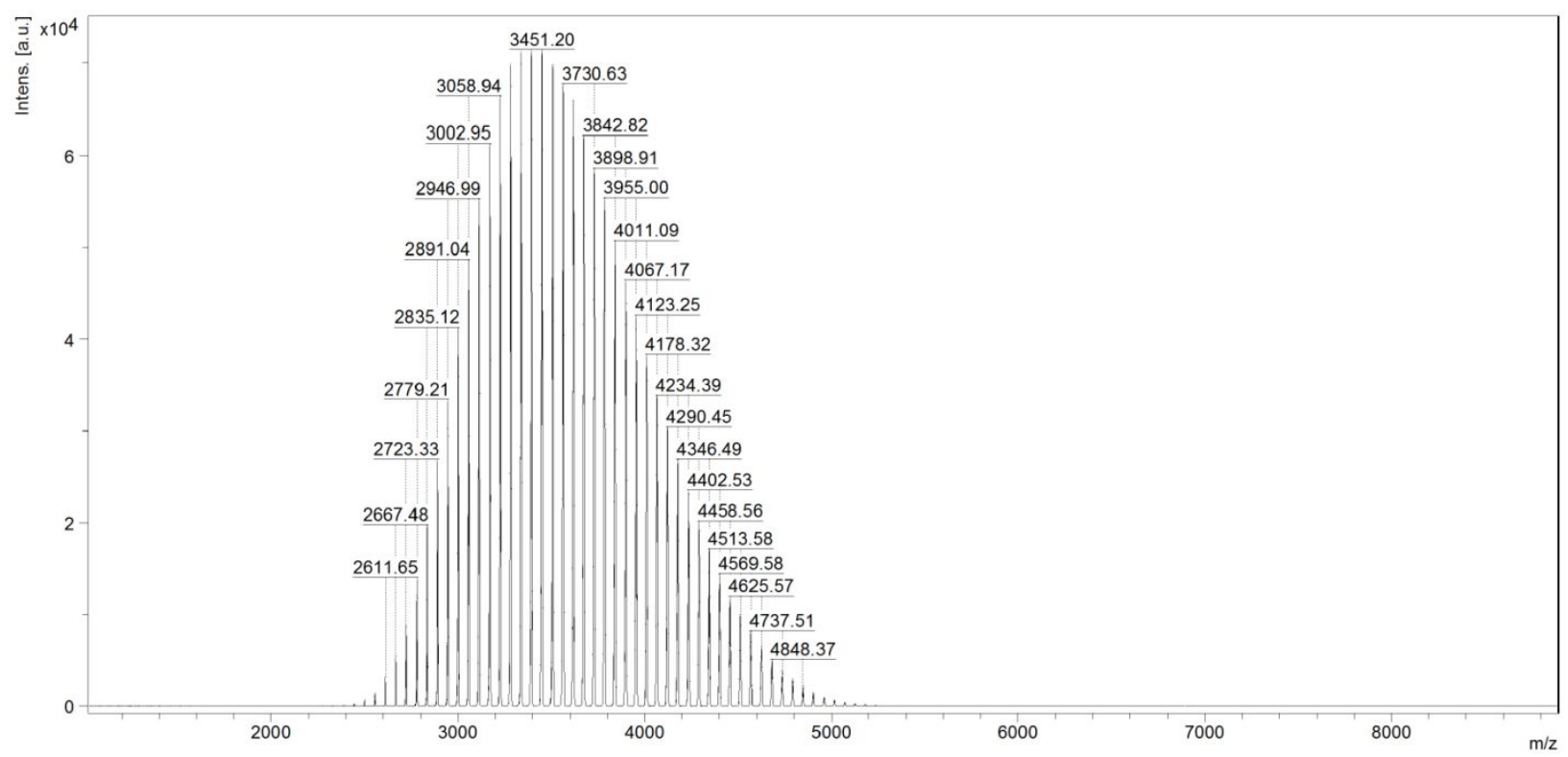


${ }^{1} \mathrm{H}$ and ${ }^{13} \mathrm{C}$ NMR Spectra $\left(\mathrm{CDCl}_{3}, 400 \mathrm{MHz}\right)$ of $\mathrm{BKB}_{2000}$.
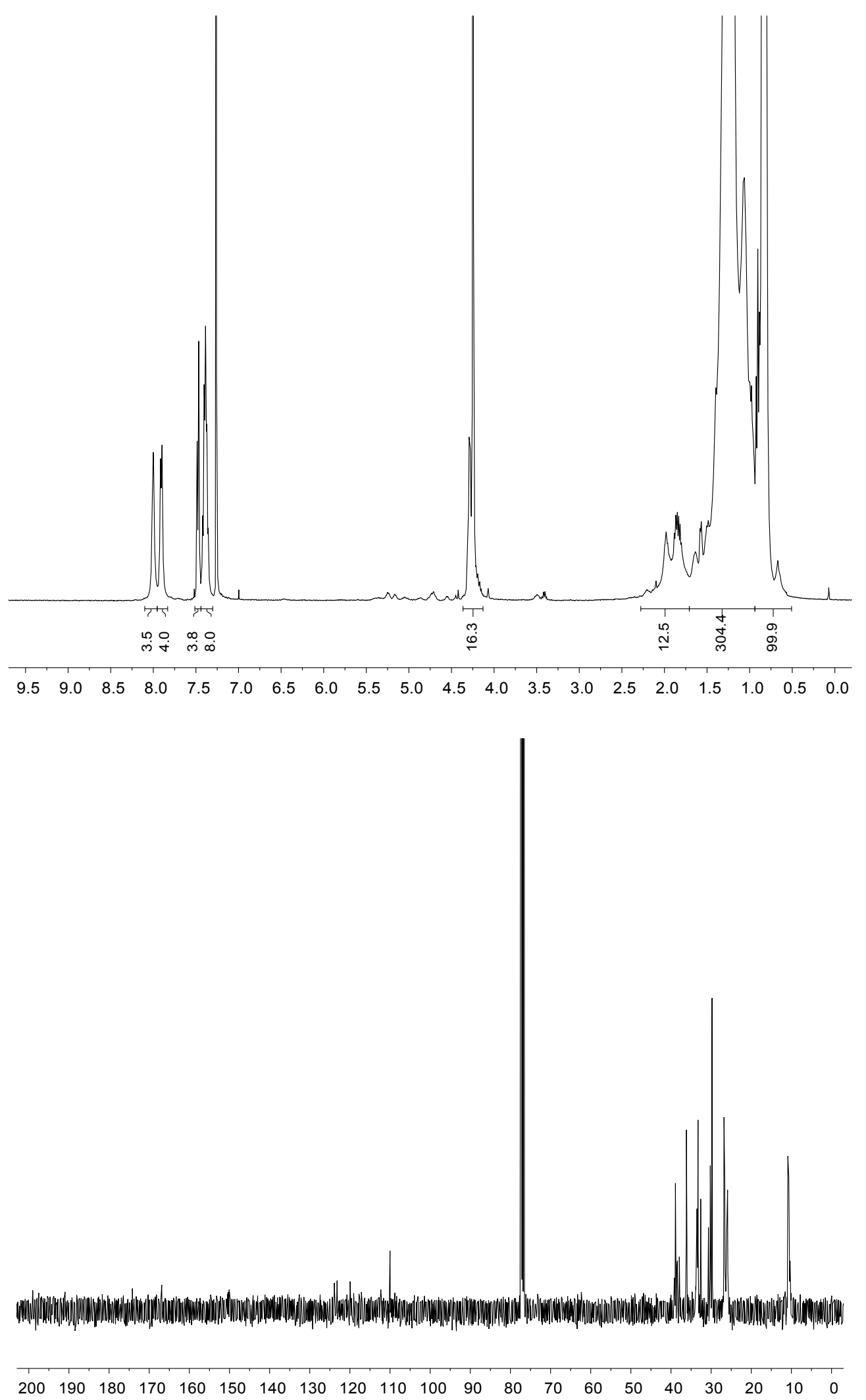


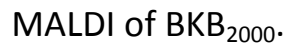

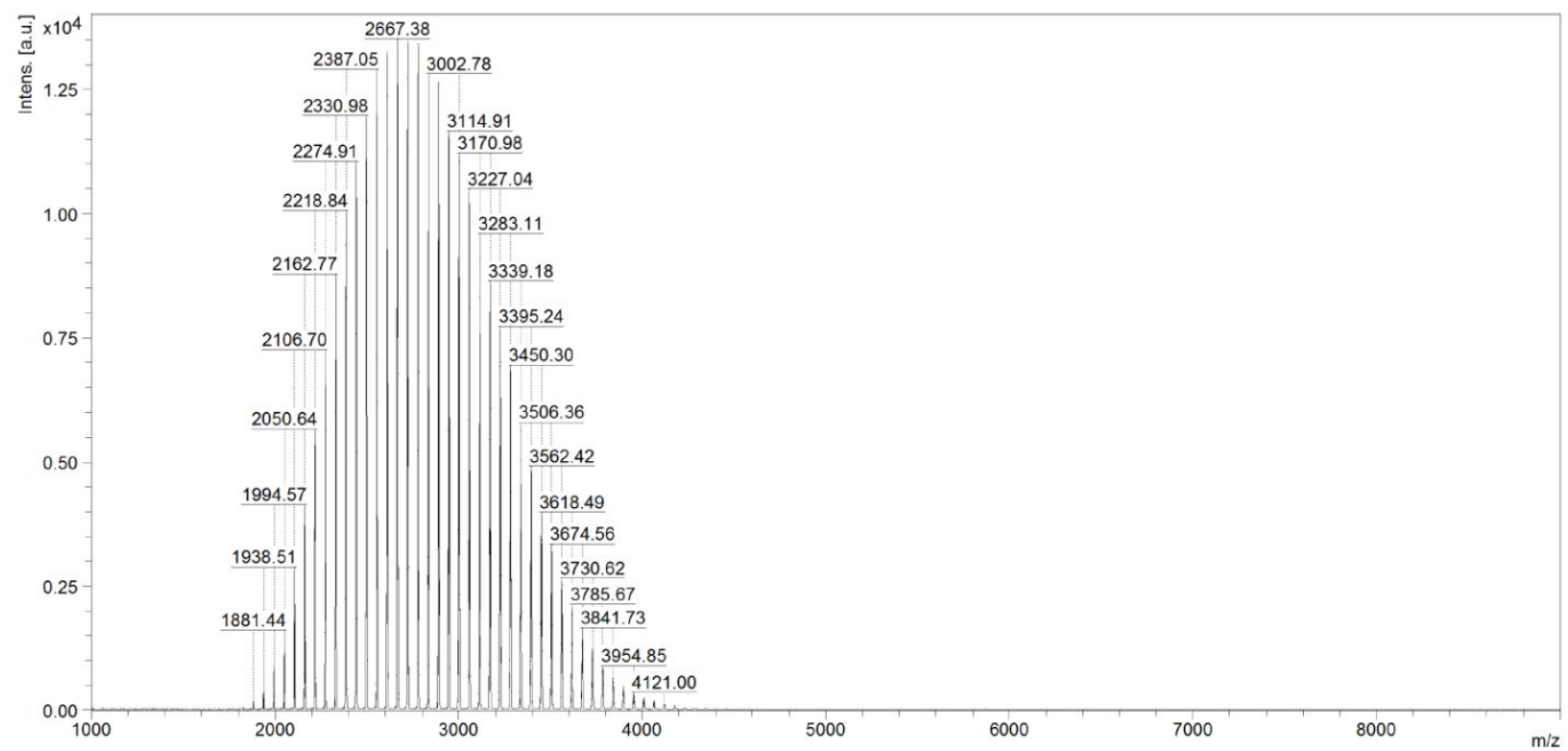




\section{References}

(1) Burnworth, M.; Tang, L.; Kumpfer, J. R.; Duncan, A. J.; Beyer, F. L.; Fiore, G. L.; Rowan, S. J.; Weder, C. Optically Healable Supramolecular Polymers. Nature 2011, 472, 334-337.

(2) Sautaux, J.; Montero de Espinosa, L.; Balog, S.; Weder, C. Multistimuli, Multiresponsive Fully Supramolecular Orthogonally Bound Polymer Networks. Macromolecules 2018, 51, 5867-5874.

(3) Petoud, S.; Bünzli, J.-C. G.; Renaud, F.; Piguet, C.; Schenk, K. J.; Hopfgartner, G. Stability and SizeDiscriminating Effects in Mononuclear Lanthanide Triple-Helical Building Blocks with Tridentate Aromatic Ligands. Inorg. Chem. 1997, 36, 5750-5760.

(4) Escande, A.; Guénée, L.; Buchwalder, K.-L.; Piguet, C. Complexation of Trivalent Lanthanides with Planar Tridentate Aromatic Ligands Tuned by Counteranions and Steric Constraints. Inorg. Chem. 2009, 48, 11321147.

(5) Scherer, G. W. Editorial Comments on a Paper by Gordon S. Fulcher. J. Am. Ceram. Soc. 1992, 75, 10601062.

(6) Bose, R. K.; Hohlbein, N.; Garcia, S. J.; Schmidt, A. M.; van der Zwaag, S. Connecting Supramolecular Bond Lifetime and Network Mobility for Scratch Healing in Poly(Butyl Acrylate) lonomers Containing Sodium, Zinc and Cobalt. Phys. Chem. Chem. Phys. 2015, 17, 1697-1704.

(7) Rowan, S. J.; Beck, J. B. Metal-Ligand Induced Supramolecular Polymerization: A Route to Responsive Materials. Faraday Discuss. 2005, 128, 43-53. 